\title{
On the Semantics of "Now" in Databases
}

\author{
JAMES CLIFFORD \\ New York University, New York, NY \\ CURTIS DYRESON \\ James Cook University, Townsville, Australia \\ TOMÁS ISAKOWITZ \\ New York University, New York, NY \\ CHRISTIAN S. JENSEN \\ Aalborg University, Aalborg, Denmark \\ and \\ RICHARD T. SNODGRASS \\ University of Arizona, Tucson, AZ
}

Although "now" is expressed in SQL as CURRENT_TIMESTAMP within queries, this value cannot be stored in the database. However, this notion of an ever-increasing current-time value has been reflected in some temporal data models by inclusion of database-resident

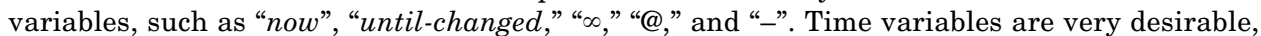
but their use also leads to a new type of database, consisting of tuples with variables, termed a variable database.

This article proposes a framework for defining the semantics of the variable databases of the relational and temporal relational data models. A framework is presented because several reasonable meanings may be given to databases that use some of the specific temporal variables that have appeared in the literature. Using the framework, the article defines a useful semantics for such databases. Because situations occur where the existing time variables are inadequate, two new types of modeling entities that address these shortcomings, timestamps that we call now-relative and now-relative indeterminate, are introduced and defined within the framework. Moreover, the article provides a foundation, using algebraic

Partial support for Curtis Dyreson and Richard Snodgrass was provided by the National Science Foundation through grants IRI-8902707 and IRI-9302244, the IBM Corporation through Contract \#1124, and the AT\&T Foundation. Partial support for Christian S. Jensen was provided by the Danish Natural Science Research Council through grants 11-9675-1 SE and 11-0061.

Authors' addresses: J. Clifford and T. Isakowitz, Information Systems Department, Leonard N. Stern School of Business, New York University, 44 W. 4th Street, New York, NY 10012; email: \{tisakowi\}@stern.nyu.edu〉; C. Dyreson, Department of Computer Science, James Cook University, Townsville, Queensland Q4811, Australia; email: 〈dyreson@cs.jcu. edu.au〉; C. S. Jensen, Department of Mathematics and Computer Science, Aalborg University, Fr. Bajers Vej 7E, Dk-9220 Aalborg Øst, Denmark; email: csj@iesd.auc.dk>; R. T. Snodgrass, Department of Computer Science, University of Arizona, Tucson, AZ 85721; email: 〈rts@cs. arizona.edu $\rangle.$ Permission to make digital/hard copy of part or all of this work for personal or classroom use is granted without fee provided that the copies are not made or distributed for profit or commercial advantage, the copyright notice, the title of the publication, and its date appear, and notice is given that copying is by permission of the ACM, Inc. To copy otherwise, to republish, to post on servers, or to redistribute to lists, requires prior specific permission and/or a fee.

(C) 1997 ACM 0362-5915/97/0600-0171 $\$ 03.50$ 
bind operators, for the querying of variable databases via existing query languages. This transition to variable databases presented here requires minimal change to the query processor. Finally, to underline the practical feasibility of variable databases, we show that database variables can be precisely specified and efficiently implemented in conventional query languages, such as SQL, and in temporal query languages, such as TSQL2.

Categories and Subject Descriptors: H.2.3 [Database Management]: Languages—data description languages (DDL), query languages; H.2.4 [Database Management]: Systemsquery processing

General Terms: Languages, Performance

Additional Key Words and Phrases: Indeterminacy, Now, now-relative value, SQL, temporal query language, TSQL2

\section{INTRODUCTION}

Now is a noun in the English language that means "at the present time" [Sykes 1964]. A variable with this name has also been used extensively in temporal relational data model proposals, primarily as a timestamp value associated with tuples or attribute values in temporal relations. Yet, the precise semantics of databases with this and other current-time variables has never been fully specified. An important goal of this article is to give a clear semantics for databases with current-time variables.

Time variables such as now are of interest and indeed are quite useful in databases, including conventional SQL databases, that record time-varying information, the validity of which often depends on the current-time value. Such databases may be found in many application areas such as banking, inventory management, and medical and personnel records. For example, in a banking application, it is necessary to record when account balances for customers are valid. Specifically, if a customer opens an account and deposits US $\$ 200$ on January 15 (in some year), the validity of that balance starts when the deposit is made and extends until the current time, assuming no update transactions are committed. Thus, on January 16, the balance is valid from January 15 until January 16; on January 17, the balance is valid from January 15 until January 17, and so on. It is impractical to update the database each day (or millisecond) to correctly reflect the valid time of the balance. Rather, it would be quite useful to be able to store a variable, such as now, to indicate that the time when a balance is valid depends on the current time. In the example, it would be recorded on January 15 that the customer's balance of US $\$ 200$ is valid from January 15 through now. Although SQL-92 [Melton and Simon 1993] has a construct CURRENT_TIMESTAMP (as well as CURRENT_DATE and CURRENT_TIME) for use in queries, one cannot store such a value in a column of an SQL table. All major commercial DBMSs have similar constructs, and impose this same restriction. The user is forced instead to store a specific time, which is cumbersome and inaccurate. This article shows how database variables such as CURRENT_TIMESTAMP can be precisely specified and efficiently implemented in conventional query lan- 
guages such as SQL-92 and in temporal query languages, while having little impact on the underlying data model.

We knew of no work on storing now in conventional databases, so we turned to the literature on temporal databases. In examining the large body of existing temporal data models, it is apparent that two different types of models have been proposed. The first type of model essentially accords with the view expressed by Reiter that a relational database can be seen as a set of ground first-order formulæ, for which there is a minimal model [Reiter 1984]. These models have either been presented as logical models directly (e.g., Clifford and Warren [1983] and Clifford et al. [1993]), or have been presented in such a way that their logical model was clear (e.g., Codd [1970]).

The second type of model deviates from this tradition. Rather, these models have been presented as a set of formulæ some of which are ground, but others of which have included one or more free, current-time variables. Chief among these current-time variables is "now" (e.g., Clifford and Croker [1987], Gadia [1988], and Clifford and Tansel [1985]), but a variety of other symbols have been used, including “-" [Ben-Zvi 1982], “ $\infty$ " [Snodgrass 1987], “@” [Lorentzos and Johnson 1988], and "until-changed" [Wiederhold et al. 1993]. As already mentioned and exemplified, the use of such variables is quite convenient and practical. Thus, these approaches have advantages at the implementation level, namely, they are space efficient and avoid the need for updates at every moment in time. However, nowhere have we found a clear exposition of temporal variables; that is, nowhere has the semantics of this type of database-a database with current-time variables, here termed a variable database-been formally specified so that the logical model represented by the database is clear. Rather, the models have relied on the choice of intuitive names for the variables to convey their meaning. This has led many to suppose that they understood their semantics. However, this reliance on intuition and lack of a clear semantics for databases with current-time variables is an unsatisfactory foundation for the development and implementation of variable databases, as it is prone to ambiguities and misinterpretations and, therefore, to errors.

In this article, we present a framework for the specification of the different semantics that may be given to variable databases, which builds on the approach introduced in Clifford and Isakowitz [1994]. In the framework, the semantics of a variable database is defined by means of an extensionalization mapping from a variable database to a fully ground data model. The actual extensionalization mappings for valid-time, transactiontime, and bitemporal databases with one or more current-time variables are given in subsequent sections. This illustrates that the framework is general enough to allow for the specification of a wide variety of semantics, an important property of a framework. It also illustrates that the framework can capture the semantics of multidimensional databases in a straightforward manner: the multidimensional extensionalization mapping 
is obtained by a simple, but coordinated, combination of the mappings for the constituent one-dimensional databases.

We also observe that the modeling capabilities of current-time variables are limited. To overcome these limitations, two new modeling entities, now-relative and now-relative indeterminate timestamps, are introduced and defined within the framework. Next, a mechanism for the querying of variable databases using existing query languages is provided. This mechanism provides added functionality, does not require changes to a query language, and is easily integrated into a query processor. It is observed that the incorporation of the notion of perspective into query languages may provide additional functionality when querying variable databases. Finally, to underline the practicality of a variable database, compact physical representations for timestamps involving current-time variables are provided. These formats can be efficiently manipulated during query processing.

\section{MOTIVATION}

To motivate the need for current-time variables in databases with timevarying data, including a solid, formal foundation for their use, this section introduces the use of such variables and explores some of the perhaps unintuitive, semantic subtleties resulting from their incorporation. Further, this section explores the limitations of current-time variables in some realistic situations.

As the meaning of current-time variables depends on whether the context is valid time or transaction time, current-time variables in valid-time and transaction-time databases are considered in isolation, followed by a short discussion of current-time variables in bitemporal databases.

\subsection{Storing Valid-Time Variables in Databases}

The valid time of a fact denotes the time(s) when the fact is true in the modeled reality [Jensen et al. 1994a; Snodgrass and Ahn 1985; Jensen and Snodgrass 1996]. In the valid-time dimension, a timestamp involving now is commonly used to indicate that a fact is currently valid [Ariav et al. 1984; Bassiouni and Llewellyn 1992; Elmasri et al. 1990; Gadia 1988; Navathe and Ahmed 1989; Sarda 1990; Tansel 1990; Yau and Chat 1991].

It is possible to explicitly record when facts are valid in both conventional SQL databases and in truly temporal (e.g., TSQL2 [Snodgrass 1995]) databases. With SQL databases, the semantics of valid time must be implemented in application programs, whereas in temporal databases, the semantics is built directly into the data model and query language. The discussion of valid time that follows is phrased in terms of temporal databases, but applies equally well to conventional databases.

As an example, suppose that a database records that Jane was on the faculty of "State University" in some particular year, for example, 1995; which year is not relevant here. Figure 1(a) shows the relevant tuple from the University's employment database (the FACULTY valid-time relation). 


\begin{tabular}{|l|l||c|}
\hline \multicolumn{3}{|c|}{ FACULTY } \\
\hline NAME & RANK & \multicolumn{2}{|c|}{ VALID TIME } \\
(from) & (to) \\
\hline
\end{tabular}

(a)

\begin{tabular}{|l|l||l|l|}
\hline Jane & Assistant & June 1 & now \\
\hline
\end{tabular}

(b)

\begin{tabular}{|l|l||c|c|}
\hline Jane & Assistant & June 1 & forever \\
\hline
\end{tabular}

(c)

\begin{tabular}{|l|l||l|l|}
\hline Jane & Assistant & June 1 & July 6 \\
\hline Jane possibly employed as an Assistant & July 6 & now \\
\hline
\end{tabular}

Fig. 1. Describing Jane's employment.

Jane started working as an Assistant Professor on June 1, as indicated by the "from" attribute. The value now, appearing as the "to" time in Jane's employment tuple, represents the (later) time when Jane will stop working for State University as an Assistant Professor. Together, the "to" and "from" attributes encode the valid time associated with the tuple. For simplicity, we assume a timestamp granularity of one day in all examples.

The informal meaning of this tuple is that Jane is a faculty member from June 1 until the current time. Thus, the result of a query that requests the current faculty members will include Jane. As the current time inexorably advances, the value of now also changes to reflect the new current time. Some authors have called this concept "until changed" [Wiederhold et al. 1993] or “@” [Lorentzos and Johnson 1988] instead of "now," but the meaning is the same.

Using the variable now in a timestamp is very convenient. To see why, suppose that instead of using the variable as the "to" time, we use a ground time, that is, a particular date. We start by recording a "to" time of June 1. Then as time advances and Jane remains an Assistant Professor, the "to" time on Jane's tuple must be updated each day to record when she worked. Hence, the "to" time would be updated to June 2, then to June 3, and so on. Although this representation is faithful to our knowledge at any point in time, having to continuously update the "to" time as time advances is impractical. It is also unclear who should do the updating, as the database has no indication of which tuples have a continuously increasing valid time and which are stable. For these reasons, it is better to use the variable now.

\subsection{Anomalies of Existing Approaches}

Here we explore four situations that illustrate shortcomings of a single variable now and thus indicate a need for additional current-time modeling entities, which we introduce in Section 4.

2.2.1 The Pessimistic and Optimistic Assumptions. Although using now is convenient, using it as the "to" time of a tuple may lead to an overly pessimistic assumption about the modeled reality. The university application introduced in the previous section provides such a situation. Specifically, it is reasonable to expect that if an employee is employed in a certain position today, that employee will also be employed in that position 
tomorrow (and the next few, following days). However, the FACULTY relation given in Figure 1(a) specifically records that Jane will not be employed tomorrow. Assume that today is July 9. Then a query asking who will be employed tomorrow (i.e., July 10) will not include Jane in the answer, since the "to" time of Jane's tuple is now, or in this case, July 9. This is overly pessimistic.

Some temporal data models avoid this problem by limiting valid time to the past, that is, "to" times before now [Gadia 1988; Tansel 1990]. For many applications, for example, the university application, this limitation is much too restrictive. Other data models have advocated using one of the special (nonvariable) valid-time values, such as forever, $\infty$, or “_" [Snodgrass 1987, 1993; Ben-Zvi 1982; Thirumalai and Krishna 1988] instead of now. These symbols (we use forever) denote the largest representable timestamp value, that is, the one furthest in the future. In SQL and in IBM's DB2, forever is about 8,000 years from the present [Melton and Simon 1993; Date and White 1990]; in our more liberal proposal, it is approximately 18 billion years from the present time [Dyreson and Snodgrass 1993a].

By using a "to" time of forever, as in Figure 1(b), we certainly avoid the pessimistic assumption, but we are now being overly optimistic. We have indicated that Jane will be employed as an Assistant Professor not only tomorrow, but until forever. To assert that Jane will be employed as an Assistant Professor until forever is most assuredly incorrect (others have also noted that a "to" time of $\infty$, or forever, has erroneous implications for the future [Navathe and Ahmed 1989]). Another indication that forever is inappropriate is that when Jane departs the University, forever must be replaced by the date of her departure; but the revised date will be a separate and much earlier time that is inconsistent with forever. Rather than having the new information refine the old information, the new information contradicts the old information. Using, instead of forever, some large, application-dependent time value earlier than forever (e.g., in the university application, the mandatory retirement date) is better than the generic forever, but is still overly optimistic. In Section 4.4, we introduce a new type of timestamp that better addresses these requirements.

2.2.2 The Punctuality Assumption. The use of now in timestamps implies a strong assumption about the punctuality of updates. For example, the tuple in Figure 1(a) states that Jane will remain an Assistant Professor until the current time. The correctness of this tuple is dependent on the correctness of the assumption that updates are made ahead of time, that is, predictively. Thus, changes in Jane's employment status and rank are assumed to conform with the punctuality assumption: "changes are recorded in the database no later than the instant they take effect."

This assumption is not often satisfied. Rather, information is often recorded after the time it became valid, but within a well-specified maximum delay [Jensen and Snodgrass 1994]. For example, when employees change status, it may be that the database is guaranteed to be updated to reflect this at most three days after the status is changed. If Jane was 
promoted on July 8, perhaps it is not until July 11 that her tuple is actually updated to reflect her correct status. With this delay, the database is known to correctly describe the miniworld only in the past, up until three days ago. Within the last three days, it can only be concluded that it is likely, or possible, that Jane is employed as an Assistant Professor. In this case, one could interpret the meaning of Jane's tuple in Figure 1(a) as of today (July 9) as shown in Figure 1(c) that intuitively illustrates the "possible" type of information that we would like to be able to record because it more accurately describes our knowledge of the miniworld. This cannot conveniently be recorded using now. Sections 4.2 through 4.4 describe a new kind of timestamp that can be used to address these issues.

2.2.3 The Problem of Now in Predictive Updates. Another problem with using the variable now as a "to" time in a tuple occurs in predictive updates where the "from" time is after the current time. Thus, the "to" time is before the "from" time, contradicting the intuition that the "to" should always be after the "from" time. To illustrate this use of now, assume that the tuple in Figure 1(a) was inserted on May 25 (i.e., the fact was recorded prior to when Jane began work). Then, during the remainder of May, the "to" time is before the "from" time.

Some data models do not allow the use of now as a "to" time when its value is before the "from" time. Instead, a special "to" time value of NULL is used in such situations [Elmasri et al. 1990; Navathe and Ahmed 1989; Yau and Chat 1991]. This value is replaced by now when the value of now exceeds the "from" time. Tuples with NULLs are ignored in queries. However, there is a subtle difficulty with this solution. Suppose that today is May 25 and we record that Jane will be an Assistant Professor from June 1 until now (or NULL in this case). We then execute a query that determines who will be employed in June barring any changes to the database between now and June. To evaluate this query, we temporarily "observe" the database from the perspective of a user in June even though today is May 25. The result should include Jane; however, Jane's tuple is ignored since it has a "to" time of NULL. In Section 4.4 we introduce a new modeling entity that addresses this shortcoming.

2.2.4 Queries and Now. When querying data that involve now, the current time must be clearly specified since the value of now depends on this time. To illustrate the kind of ambiguity that can result from unclear specification of the current time, assume that today is July 9 and that our database is given as in Figure 1(a). Then, consider the query, "Will we agree on July 13 that Jane was employed on July 11?" Suppose that now is interpreted to refer to the time at which the query is asked, in this case July 9 . Then Jane will not be employed on July 11 and so we would answer "no." But now could be interpreted as the time mentioned in the query about which we were asked to agree, in this case July 13 . Then Jane will be employed on July 11 and so we would answer "yes."

Another source of ambiguity is that the constant evolution of the currenttime variable now appears to cause the "same" query to return different 


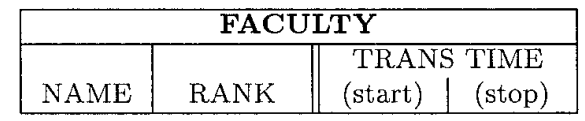

(a)

\begin{tabular}{|l|l||l|l|}
\hline Jane & Assistant & June 1 & now \\
\hline
\end{tabular}
\begin{tabular}{|l|l||l|l|}
\hline Jane & Assistant & June 1 & forever \\
\hline
\end{tabular}

Fig. 2. Describing Jane's employment in a transaction-time relation.

results when evaluated at different times, even if no updates have occurred. For instance, consider the query, "Is Jane employed on July 11?" This simple query asked on July 10 will yield one answer ("no"), but if we ask the query on July 12 we will receive a quite different answer ("yes"). Hence, the querying of variable databases introduces new semantic subtleties, not found when querying nonvariable databases.

\subsection{Storing Transaction-Time Variables in Databases}

The transaction time of a database fact denotes the time(s) when the fact is (logically) current in the database [Snodgrass and Ahn 1985; Jensen et al. 1994a]. It is an orthogonal concept to valid time, in that it concerns the evolution of the database, as opposed to the enterprise being modeled. The use of current-time relative variables in transaction-time databases introduces a different set of problems.

Whereas a valid-time timestamp is generally supplied by the user, a transaction-time timestamp, an interval from a "start" to a "stop" time, is supplied automatically by the DBMS during updates. Insertions initialize the "start" time to the "current time" and the "stop" time to now. ${ }^{1}$ Deletions are accommodated by changing "stop" times of now to the "current time." Hence, deletion is logical. The information is not physically removed from the relation; rather, it is tagged as no longer current by having a "stop" time different from now. Updates may be considered combinations of deletions and insertions.

As an example, consider the transaction-time relation in Figure 2(a). The distinct semantics of transaction time yields a different interpretation of this relation as compared with the one shown in Figure 1(a). The "start" time of June 1 indicates that this tuple was stored in the database on June 1 ; that is, we first became aware that Jane was an Assistant Professor on that date. The value of now for the "stop" attribute indicates that the database still records that Jane is an Assistant Professor; that is, the fact is current. If we learn on July 10 that Jane left State University and thus (logically) delete the fact, this is reflected by changing the "stop" time to July 10.

The problem with using a variable called now in transaction time is that the name "now" obscures the use of the variable. Strictly speaking, it

\footnotetext{
${ }^{1}$ The transaction processing system must also ensure that the "start" times of tuples are consistent with a serialization order of their respective transactions.
} 
implies that every current tuple was deleted by the current transaction! In Figure 2(a), if the current time is July 9, then a strict interpretation of a "stop" time of now suggests that the "stop" time is July 9. This is not what was intended.

As with valid time, some data models address this problem by using forever (also called $\infty$ or "-") instead of now, as shown in Figure 2(b) [Ben-Zvi 1982; Bhargava and Gadia 1989; Snodgrass 1987; Thirumalai and Krishna 1988]. Using this large value, we immediately encounter difficulties. The strict interpretation of this tuple is that some transaction executing a (very) long time in the future will logically delete this tuple from the relation. In the meantime, it will remain in the database. If, on July 10, it becomes known that Jane has left State University, then we logically delete this tuple by changing the "stop" time to July 10. Such a change is inconsistent with the previous "stop" time. Put differently, in this scenario the database first records that we believe that Jane is an Assistant Professor from June 1 until "forever." The subsequent update then contradicts this belief by saying that it is only from June 1 until July 10 that we believe Jane is an Assistant Professor.

There is a more fundamental problem with forever in transaction time. By the semantics of transaction time, storing future transaction times is equivalent to predicting future states of the database, which is a highly problematic proposition. With no crystal ball at hand, it is customary to avoid predictions and require that the right endpoint of every interval be less than or equal to the current time. To distinguish "now" in transaction time from "now" in valid time, we propose in this paper to adopt the name "until changed" for the former and provide a precise semantics for its interpretation.

\subsection{Variables in Bitemporal Databases}

Bitemporal databases support both valid time and transaction time [Jensen et al. 1994a]. The confusion that has arisen in a number of bitemporal data models between the use of the same variable in both dimensions was a prime motivation for the semantic framework that we present in the following. In order to allow for a completely general treatment of the semantics of these variables, we use a different variable in each dimension. In Section 6 we show how the concept of a reference time can coordinate the interaction between the current-time variables in both time dimensions.

\section{SEMANTIC FRAMEWORK}

In order to provide a precise semantics for databases with current-time variables, we propose a semantic framework for defining the meaning of databases with variables in terms of databases of a fully extensional temporal data model. Databases in this latter model are fully ground; that is, they do not admit variables. Although the model is not suitable for the implementation of temporal databases, it is well suited for capturing the semantics of variable databases. 


\subsection{The Temporal Universe}

The framework developed in the following includes three distinct time dimensions, each with its own temporal universe. The framework requires the existence of well-defined mappings between these universes. Although this requirement does not preclude the possibility of different granularities for the universes, we choose to avoid such diversions and instead use a single, underlying granularity. This yields an homogeneous treatment of all time dimensions and their relationships.

Since most database researchers have adopted the view that valid time in a database is best viewed as discrete, and every database transaction model that we are aware of has this property, we also adopt a discrete model of time. Let $\mathscr{T}_{Z}$ to be the totally ordered set $\{\ldots,-2,-1,0,+1,+2, \ldots\} \cup$ $\{\perp, \top\}$, where $\perp$ (bottom) and $\top$ (top) are two distinguished elements, which intuitively correspond to $-\infty$ and $\infty$, respectively. The total order $<_{\mathscr{T}_{Z}}$ on $\mathscr{T}_{Z}$ is the normal order on integers extended so that $\perp$ and $T$ are a bottom and a top element, respectively; that is,

(1) for any two integer elements $z$ and $z^{\prime}$ of $\mathscr{T}_{Z}, z<_{\mathscr{T}_{Z}} z^{\prime}$ if $z<z^{\prime}$ (as integers); and

(2) for any element $z$ in $\mathscr{T}_{Z}, \perp \leq_{\mathscr{T}_{Z}} z \leq_{\mathscr{T}_{Z}} \top$.

The only requirement on our temporal universe $\mathscr{T}$ is that it has the same order structure as $\mathscr{T}_{Z}$. That is, $\mathcal{T}$ can be any ordered set of order type $1+$ $* \omega+\omega+1$ [Fraenkel et al. 1973, p. 128]. ${ }^{2}$ For example, in most of the examples in this article we chose $\mathscr{T}$ to be the ordered set of days, extended infinitely into the past and the future, with added elements $-\infty$ and $\infty$.

In addition to the concepts of valid time and transaction time, we introduce a third time, reference time, to represent the relationship between a temporal database and the "real world" time at which it is viewed. Thus, three temporal universes are required in the framework, namely, the reference-time, the valid-time, and the transaction-time universe, and it may be desirable or convenient to restrict them to some subset of $\mathcal{T}$. Therefore, let

$\mathscr{T}_{R T} \subseteq \mathscr{T}$ denote the reference-time universe of our database,

$\mathscr{T}_{V T} \subseteq \mathcal{T}$ denote the valid-time universe of our database, and

$\mathscr{T}_{T T} \subseteq \mathcal{T}$ denote its transaction-time universe.

\subsection{Important Times}

Throughout our discussion of variable databases and queries on these databases, five distinct times surface repeatedly. The first of these is called initiation. It is relative to a specific relation and denotes the transaction

\footnotetext{
${ }^{2}$ The ordinal 1 stands for the order type of a one-element set. The ordinal $\omega$ is the ordinal type of an enumerated set, for example, the nonnegative integers with their familiar ordering: 0,1 , $2,3, \ldots ;$ whereas $* \omega$ represents the inverse of that ordinal, that is, the type of . . , 3, 2, 1,0 . Thus, $1+* \omega+\omega+1$ stands for the following ordinal type "an element before . . , 3, 2, 1, 0 , $1,2,3, \ldots$, followed by another element." The appearance of one zero instead of two (. . ., 3, $2,1,0,0,1,2,3, \ldots)$ does not change the ordinal.
} 
time when that relation was created. To simplify the discussion that follows, we assume that all relations are created at the same time, denoted by $t_{0}$. Once created, we assume that the database schema never changes (schema versioning [Roddick 1992] is orthogonal to most of the issues discussed in this article).

The second important time, which is new to most readers, is the reference time. The reference time is the time of the database observer's "frame of reference," denoted by $r t_{*}$. Reference time is a term analogous to the indices or "points of reference" in intensional logic [Montague 1974], and discussed more recently in the context of valid-time databases [Finger 1992]. The reference time facilitates a kind of "time travel" by means of which we may observe the database at times other than the present.

A related time is the query time, or current transaction time, denoted by $t_{\text {current }}$. It is the time at which a query is processed. The reference time $r t_{*}$ and current time $t_{\text {current }}$ are related, but distinct. In general, $t_{\text {current }}$ is the time at which a query is initiated, and $r t$ * is the time at which the user "observes" the database. In many queries, the reference time and the query time are the same. But the user may choose to observe the database from a previous perspective; for this kind of query, the reference time is earlier than the query time. For example, if today is July 9 and we wish to observe the database from the perspective of a week ago, then $t_{\text {current }}=$ July 9 and $r t_{*}=$ July 2 .

The final two times of special interest are the valid timeslice time $v t_{*}$ and the transaction timeslice time $t t_{*}$. These times are important in this article because, for expository purposes, we focus exclusively on various timeslice queries. The valid and transaction timeslice times could both be an instant, an interval, or a set of instants or intervals. The valid timeslice time(s) specifies the real-world time about which information is wanted, whereas the transaction timeslice time(s) is the time(s) during which information must be current in the database in order to be of interest for a query. For the example queries given in this article, it is advantageous to choose instants (as opposed to intervals) as the valid timeslice and transaction timeslice times. Later, we see that, although these times are distinct concepts, there are important relationships between the valid timeslice time, the transaction timeslice time, and the reference time.

To illustrate the distinction among these five times, let us consider an example. A temporal database for recording employment information is created on January 11 (again, the particular year is immaterial). Today (which we assume is July 9), the director of the personnel department investigates an apparent discrepancy reported by a coworker a week earlier, while using the database on July 2. The coworker discovered that the database had mistakenly recorded on June 27 that an employee had been hired two weeks earlier, on June 13. The five times in this example are as follows.

(1) $t_{0}$ is January 11 , the day of the creation of the database;

(2) $r t_{*}$ is July 2 , the day when the problem was observed; 
(3) $t_{\text {current }}$ is July 9 , the day the personnel department director investigates the database;

(4) $v t_{*}$ is June 13, the real-world day of the problematic information; and

(5) $t t_{*}$ is June 27 , the day for which we are interested in what was recorded as current information in the database.

By using a reference time of July 2, the director can view the identical database state in existence when the coworker discovered the discrepancy. If a reference time of June 20 had been used instead, it is possible that no discrepancy would have been found, because that date was well before $t t_{*}$. Although purposely contrived, this example highlights the differences among the five times. Having made this point, this example is not used in the remainder of this article.

We have the following constraints on these five times.

$-\perp \leq t_{0} \leq t t_{*} \leq t_{\text {current }} \leq \top$.

$-\perp \leq r t_{*} \leq \top$.

$-\perp \leq v t_{*} \leq \top$.

Note that $r t_{*}$ is not bound by $t_{\text {current }}$. This provides the ability to ask "hypothetical now" queries, that is, from the perspective of a future valid time (i.e., ten years from now). Such an example is given later in Section 6.2.

\subsection{Extensional and Variable Database Levels}

It is useful to view the semantics of temporal databases with variables within the context of a two-level framework. This section develops such a framework by first presenting the levels of a theoretical framework. Then this framework is augmented, motivated by the practical concerns of easily extending existing data models to admit databases with variables, such as now, with minimal impact on existing query languages and query processing engines.

A relational database consists of a set of relations, where each relation is a set of tuples. Each tuple in a relation has a number of application-specific attribute values. Temporal databases extend this view by incorporating the temporal aspects of data using special attributes, termed timestamps. These are explored further next.

In our model of time from the previous section, time instants (or just instants for short) are points in time and intervals are sequences of temporally consecutive points. (Indeed, when time is discrete, intervals are merely shorthand for a finite, or countably infinite, set of instants.) Intervals may be uniquely described by two bounding instants, termed the starting and terminating instants.

Each of the valid and transaction times of data may be recorded by associating a single time interval or a single instant with each tuple. Interval timestamps are very convenient at the conceptual and implementation levels, as they are compact and can represent information about a potentially large number of times in a single tuple. Thus, following a range 


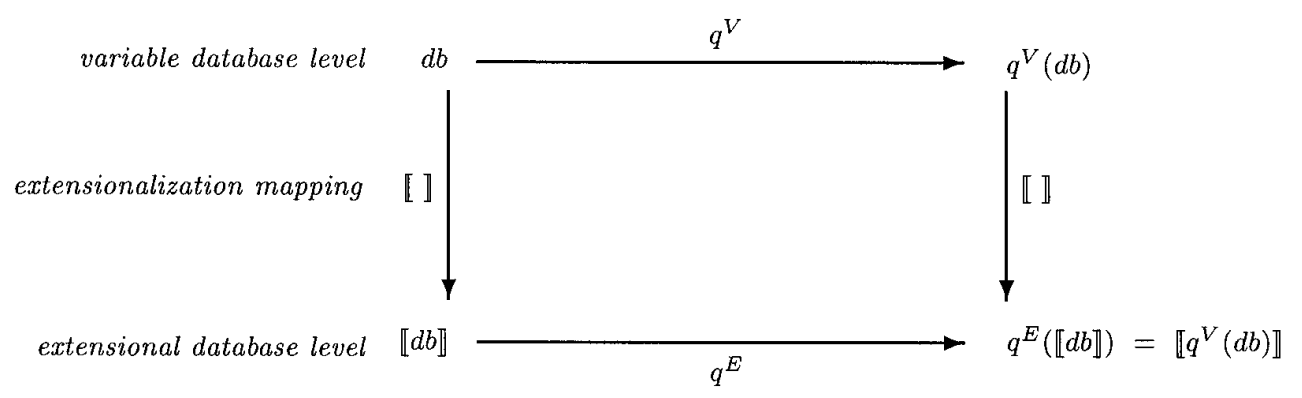

Fig. 3. Relationship between variable and extensional databases.

of temporal data models, we assume interval timestamps at the variable database level.

We employ specific names for the timestamp attributes that encode the time intervals of tuples. For valid-time intervals, the starting instant is recorded by an attribute "from" and the terminating instant is recorded by an attribute "to"; see Figure 1 for an example. For transaction-time intervals, we use "start" and "stop," as in Figure 2. In a variable database, the values of the timestamp attributes in any tuple are extended to permit instances of one or more current-time variables, as discussed earlier. Figure 1(a) gives a simple variable database with only one tuple.

Moving to the extensional level, tuples also have timestamp attributes. However, there are three key differences. First, no variables are allowedthe extensional level is fully ground. Second, timestamps are instants rather than intervals. Third, an extensional tuple has one additional temporal attribute, called a reference time attribute. Later in this article we describe the importance of reference time to the meaning of tuples. For now, it may be thought of as representing the time at which a meaning was given to the temporal variables in the original tuple.

Whereas the variable-database level offers a convenient representation that end-users can understand and that is amenable to implementation, the mathematical simplicity of the extensional level supports a rigorous treatment of temporal databases in terms of first-order logic. A theoretical framework for providing a logical interpretation or "meaning" for a particular variable database (i.e., a "translation" from variable to extensional level), may be based on a homomorphic mapping from variable-level databases to extensional-level databases [Clifford and Isakowitz 1994]. This mapping is termed an extensionalization, and is denoted $\llbracket \rrbracket$. In addition to giving the semantics of variable databases, the framework also provides a means for checking the correctness of query languages over variable databases. This is illustrated in Figure 3 and explained using an example.

The top of the figure, labeled the variable database level, represents a database model that allows the use of temporal variables in timestamps of tuples. At the top left, we see a particular variable database $d b$. The tuple 〈Jane, Assistant, [June 1, now] (with now being a variable) from Figure 1 (a) is an example. A query $q^{V}$ is applied to this database, resulting in 
another variable database, $q^{V}(d b)$. Let $q^{V}$ be "List the faculty on June 15," and assume this query is evaluated on June 27. The result is then $\{\langle$ Jane, Assistant $\rangle$.

The bottom of the figure, labeled the extensional database level, represents our fully extensional temporal data model, whose semantics is well-specified in the standard tradition of a first-order logical framework. Developing a query language in this extensional model is relatively straightforward, due to the model's simplicity. In contrast, developing a query language for a more complex variable-level data model is error prone. The framework can be used for checking the correctness of variable-level query constructs. Specifically, variable-level query constructs must commute with the corresponding extensional-level query constructs, as indicated in the figure: $q^{E}(\llbracket d b \rrbracket)=\llbracket q^{V}(d b) \rrbracket$.

A particular extensionalization mapping from the top level to the bottom level is defined in order to specify the semantics of variable databases. As tuples at the variable database level are independent of each other, an extensionalization mapping may treat each tuple in isolation.

Continuing the example, an extensionalization mapping ${ }^{3}$ may map 〈Jane,

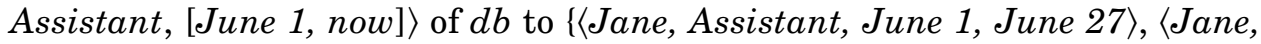

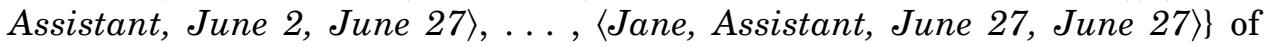
$\llbracket d b \rrbracket$. In the extensional database level, valid-time tuples are associated with two (instant) timestamps. The first timestamp (e.g., June 1) is the instant when the fact was valid (the interval is deconstructed into its component instants), and the second time (e.g., June 27) is the reference time. The extensional-level version of the query then selects the tuple from this set that has a valid time of June 15 , giving as result $q^{E}(\llbracket d b \rrbracket)=\{\langle$ Jane, Assistant, June 27)\} (again omitting the valid time). Finally, applying the extensionalization mapping to the variable-level query result, $\{\langle$ Jane, Assistant $\rangle$, yields $\llbracket q^{V}(d b) \rrbracket=\{\langle$ Jane, Assistant, June 27 $\rangle$. The diagram thus commutes for the sample database and query. Section 4 and subsequent sections provide a thorough coverage of extensionalization.

We are concerned in this article with the practical use of variable databases. In particular, we are interested in how to extend existing data models and query languages with the ability to allow current-time variables, with as little impact as possible on their conceptual model and their associated query-processing engines. This is consistent with the philosophy of the designers of the proposed temporal extension to SQL-92, termed TSQL2 [Snodgrass 1995]. Thus, we next augment the theoretical framework as shown in Figure 4.

Figure 4 shows that at the variable database level, the database is mapped to an intermediate stage, in which the tuples contain timestamps but no variables, by applying a bind ${ }^{V}$ operation, which is discussed further in the following. The various existing temporal data models, including SQL-92 and TSQL2, that do not permit variable timestamps in their

\footnotetext{
${ }^{3}$ Here, we consider only a reference time of June 27. In the discussion to follow, $\llbracket$ takes an optional subscript. We omit these subscripts here to simplify the discussion.
} 


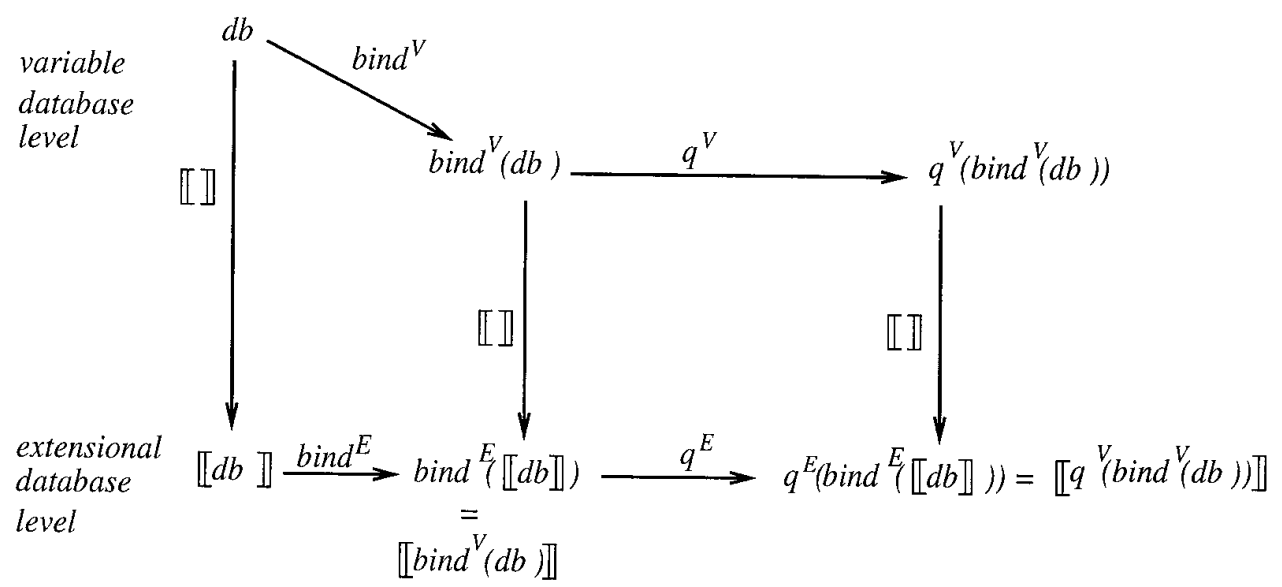

Fig. 4. Preprocessing of variable-level databases.

databases belong to this stage. By mapping variable-level databases to this intermediate stage, it is possible to reuse existing-or proposed-query engines to query variable databases. This is the motivation for augmenting the framework to permit preprocessing of variable databases before querying them.

The preprocessor substitutes each instance of a variable with a specified time, effectively "binding" the variables in a variable database (as discussed in Section 7, this occurs during query evaluation on a per-tuple basis). The bind operator bind ${ }^{V}$ maps a database from the variable to the intermediate level, upon which it is queried with a variable-level query $q^{V}$. The correctness of this mechanism is ensured by providing extensionallevel counterparts to the preprocessor and to the queries, bind ${ }^{E}$ and $q^{E}$, respectively, and by demonstrating that the preceding diagram commutes.

To exemplify, as before let the database $d b$ contain the single tuple in Figure 1(a), let the query $q$ be "List the faculty on June 15," and let the current date be June 27. To evaluate $q$ on $d b$, we first bind the variable now to the reference time, June 27. The result is bind ${ }^{V}(d b)=\{\langle$ Jane, Assistant, [June 1, June 27] $\}$. The query can then be evaluated on this relation using simple (and already accepted) methods, resulting in $q^{V}\left(\operatorname{bind}^{V}(d b)\right)=$ $\{\langle$ Jane, Assistant $\rangle$.

By defining queries $q^{V}$ at the variable-level in this way, as a composition of a binding operator and a ground query, we conceive a framework where the commutativity of the diagram shown in Figure 3 holds. Although the particular binding we exhibit here is simple, Section 2 showed this need not be the case for all temporal databases, particularly not for bitemporal databases. For this reason, we need a precise semantics, provided by the extensionalization mapping and the extensional counterpart to the query operators, and a correctness criterion, that is, the commutativity requirement. 


\section{VALID-TIME DATABASES}

Here we present a semantics for variable valid-time databases by specifying mappings from the variable to the extensional level. We initially consider the extensionalization mappings for databases with ground timestamps and timestamps with the variable now. In order to address the shortcomings identified in Section 2, we also introduce additional currenttime modeling entities. Specifically, we consider now-relative timestamps that allow for positive and negative displacements from now. Next, we introduce so-called indeterminate time values that may be used in timestamps to indicate imprecise times. This leads to a further generalization of now-relative instants to now-relative indeterminate instants, which are values that are imprecise as well as current-time relative. The section concludes with an illustration of the querying of variable databases.

\subsection{Extensionalization of Valid-Time Tuples with Now}

We first consider the extensionalization of tuples with ground timestamps. To do this, it is convenient to start by defining the meaning, or denotation, of the ground component in a timestamp. As other timestamp values are introduced, their denotations are also defined.

Definition 4.1 [Denotation of a Time Instant]. The denotation of a valid-time instant $t$ at a particular reference time $r t_{*}$, written $\langle\langle t\rangle\rangle_{r t_{*}}$, is defined as follows.

$$
\langle\langle t\rangle\rangle_{r t *}={ }_{d f} t
$$

In general, to map a ground valid-time tuple, that is, a tuple without variables, to the extensional database level, the tuple is expanded into a set of tuples, one for each time instant in its associated timestamp. Let us consider first the extensionalization of a ground tuple at a particular reference time. We use the notation $\llbracket T \rrbracket_{r t_{*}}$ to denote the extensionalization of tuple $T$ at a reference time of $r t_{*}$.

Definition 4.2 [Extensionalization of a Tuple at an Instant]. The extensionalization of a ground tuple $T$ of the form $T=\left\langle X,\left[v t_{1}, v t_{2}\right]\right\rangle$, where $\left[v t_{1}, v t_{2}\right]$ denotes the set of times $\left\{v t \mid v t_{1} \leq v t \wedge v t \leq v t_{2}\right\}$, at reference time $r t$ * is defined as follows.

$$
\llbracket T \rrbracket_{r t^{*}}={ }_{d f}\left\{\left(X, v t, r t_{*}\right) \mid v t \in\left[\left\langle\left\langle v t_{1}\right\rangle\right\rangle_{r t^{*}},\left\langle\left\langle v t_{2}\right\rangle\right\rangle_{r t^{*}}\right]\right\} .
$$

Note that each tuple at the extensional level is tagged with the reference time.

To exemplify, assume that the academic career of Jane at State University is given by the tuple $T=\langle$ Jane, Assistant, [June 3, June 9] $\rangle$. The extensionalization mapping of this tuple at time June 6 , that is, $\llbracket T \rrbracket_{\text {June } 6}$, consists of seven tuples: $\{\langle$ Jane, Assistant, June 3, June 6 $\rangle$, 〈Jane, Assistant, 
June 4, June 6〉, ..., 〈Jane, Assistant, June 9, June 6〉\}. Recall also the sample mapping given in Section 3.3.

Definition 4.3 [Extensionalization of a Tuple at an Interval]. In the extensionalization mapping, a reference time interval may be used rather than a single reference time. The extensionalization of the tuple $T$ over the reference time interval $\left[r t_{1}, r t_{2}\right]$ is defined as follows.

$$
\llbracket T \rrbracket_{\left[r t_{1}, r t_{2}\right]}={ }_{d f} \cup{r t * \in\left[r t_{1}, r t_{2}\right]} \llbracket T \rrbracket_{r t_{*}} .
$$

Definition 4.4 [Extensionalization (Complete)]. The complete meaning or extensionalization of a tuple $T$, denoted $\llbracket T \rrbracket$, is simply the extensionalization of $T$ over all reference times, that is, $\cup_{r t_{*} \in \mathcal{T}_{R T}} \llbracket T \rrbracket_{r t_{*}}$. Equivalently, the general meaning or extensionalization of a tuple $T$ is: $\llbracket T \rrbracket={ }_{d f} \llbracket T \rrbracket_{[\perp, \top]}$.

We have found that a two-dimensional graphical notation makes validtime concepts easier to grasp. In the visualization, reference time corresponds to the $X$-axis and valid time corresponds to the $Y$-axis. The graphical representation is a plot of the tuple at the extensional database level. Each cell in the plot stands for a particular reference time $R T$ and valid time VT combination. The cells corresponding to the temporal coordinates of tuples in the extensional set of tuples are shaded, indicating when a tuple is valid relative to the reference time of an observer. Even though our underlying model of time is discrete, we treat each cell as a region rather than a point since this results in a better visualization. Several tuples may be plotted in the same graph by using different cell colors or patterns. The key, shown below the graph, indicates the explicit attribute values of the corresponding tuples. Variations of these graphs have been independently explored [Jensen et al. 1994b; Jensen and Snodgrass 1992; Clifford and Isakowitz 1994].

As an example, Figure 5(a) shows the extensionalization of Jane's employment tuple from before for a sequence of reference times, June 1 through June 11, that is, $\llbracket T \rrbracket_{[J \text { Jue 1, June 11] }}$. The figure illustrates that the valid time of this tuple is reference-time invariant; that is, it is independent of the reference time. So for a tuple with a valid-time interval but without variables, it does not matter at what time the tuple is observed-it is always valid over exactly the same interval.

The meaning of a tuple with the variable now, however, is not referencetime invariant. The denotation of now makes this dependence explicit.

Definition 4.5 [Denotation of Now]. The denotation of the current-time variable now at a particular reference time $r t *$ is defined as follows.

$$
\langle\langle n o w\rangle\rangle_{r t *}={ }_{d f} r t_{*}
$$

This is precisely how reference time enables us to "materialize" variables in the extensional level. Although variables per se are not permitted at the extensional level, a valid-time tuple does vary with reference time. With 


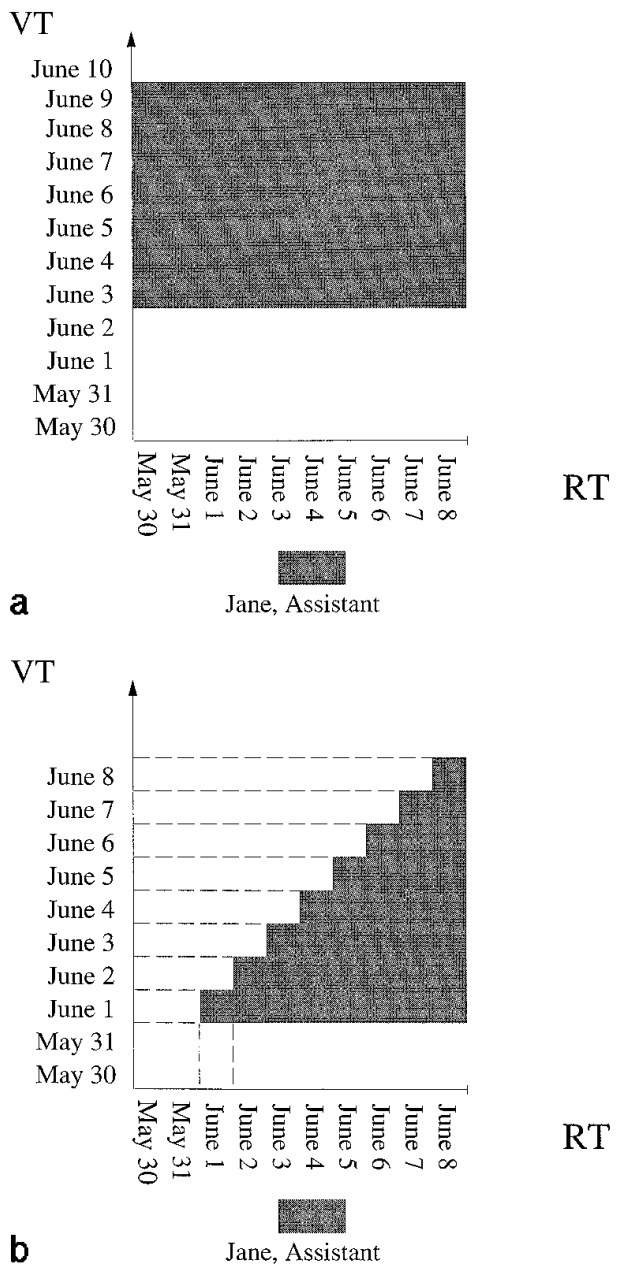

Fig. 5. Graphical representation of extensionalization of a valid-time tuple: (a) ground valid-time tuple; (b) valid-time tuple with variable.

this additional timestamp value, the extensionalization of a tuple with now as the "to" or "from" time is still given by Definition 4.2.

As an example, assume that the academic career of Jane at State University is given by the tuple $T=\langle$ Jane, Assistant, [June 1, now]〉. Figure 5(b) visualizes the extensionalization of this tuple for every reference time between May 30 and June 8. Note that before June 1 the empty interval is depicted in the figure. This is because a timestamp with a "to" time that is before the "from" time denotes the empty interval. This situation occurs prior to June 1 . The valid-time region in the figure is "stair-shaped" since the extensionalization of a tuple with variables is dependent on the time at which we observe the tuple. The stair shape is a result of the constraint that the "to" time in the valid-time interval is bound to the reference time. 
It is our contention that all other valid-time current-time variables currently in use (e.g., “@” [Lorentzos and Johnson 1988] and until-changed [Wiederhold et al. 1993]) have the same meaning as now. Thus having covered existing variables, we now proceed by proposing new timestamps that address the shortcomings of now discussed in Section 2.

\subsection{Now-Relative Instants}

In this section we introduce a new type of timestamp, called a now-relative instant, that adds flexibility to the variable now. A now-relative instant generalizes the variable now by allowing an offset from this variable to be specified. Now-relative times were first introduced in transaction time for vacuuming [Jensen and Mark 1990].

With now-relative instants, we have a means of more accurately recording our knowledge of Jane's employment with State University. For example, it may be that changes in hirings at State University are recorded in the database only three days after they take effect. Assuming that Jane was hired on June 1, we can accurately record our definite knowledge of her employment in the tuple 〈Jane, Assistant, [June 1, now - 3 days]〉. This tuple states that Jane was an Assistant Professor from June 1 and until three days ago, but it contains no information about her employment as of, for example, yesterday.

A now-relative instant thus includes a displacement, which is a (signed) span, from now. In the given example, the displacement is minus three days. The extensionalization of tuples with now-relative instants is formalized as follows.

Definition 4.6 [Denotation of a Now-Relative Instant]. The denotation of a now-relative instant, now OP $n$ days, where OP $\in\{+,-\}$, at a particular reference time $r t *$ is defined as follows.

$$
\langle\langle\text { now OP } n \text { days }\rangle\rangle_{r t *}={ }_{d f}\langle\langle n o w\rangle\rangle_{r t *} \text { OP } n \text {. }
$$

Even with this additional timestamp value, the extensionalization of a valid-time tuple is still given by Definition 4.2 .

Although now-relative instants allow us to relax the otherwise close coupling between valid and transaction time found in the punctuality assumption, now-relative instants still suffer from making a pessimistic assumption. The use of now - 3 days in the first example is an ultrapessimistic view of the future. Jane would not even be employed now since her employment terminates three days prior to now. To address this potential shortcoming, we next introduce the notion of indeterminate timestamp values.

\subsection{Indeterminate Timestamp Values}

It turns out that support for valid-time indeterminacy [Brusoni et al. 1995; Dutta 1989; Gadia et al. 1992; Kurutach and Franklin 1993] can also alleviate the shortcomings of now and now-relative instants. This section 


\begin{tabular}{|l|r||r|r|}
\hline \multicolumn{3}{|c|}{ FACULTY } \\
\hline NAME & RANK & (from) & VALID TIME \\
(to $)$
\end{tabular}

(a)

\begin{tabular}{|l|l||l|l|}
\hline Jane & Assistant & June 1 & July $31 \sim$ forever \\
\hline
\end{tabular}

(b)

\begin{tabular}{|l|l||l|l|}
\hline Jane & Assistant & June 1 & July $31 \sim$ January 1, 2028 \\
\hline
\end{tabular}

Fig. 6. Using indeterminate timestamps for recording Jane's appointment.

introduces indeterminate timestamp values for ground timestamps. The next section extends this treatment to indeterminate timestamps with variables.

Sometimes, the time when an event occurred is known only imprecisely. For instance, we may know that an event happened "sometime in June 1993," which is an imprecise period of 30 days. An indeterminate instant is the time of an event, which is known to have occurred, but exactly when is unknown [Dyreson and Snodgrass 1993b; Dyreson 1994].

The times when the event might have occurred is called the period of indeterminacy and is delimited by a lower and an upper bound (e.g., the event occurred sometime between June 1 and June 30). An indeterminate instant could have an associated probability distribution that gives the probability that the event occurred for each time in the period of indeterminacy. For the purposes of this article, we ignore the probability information: every indeterminate instant is treated as though it has a distribution that is missing [Dyreson 1994]. A determinate instant may be thought of as an indeterminate instant, with identical lower and upper bounds. An indeterminate interval is an interval bounded by indeterminate instants.

By using indeterminate instants, we can more accurately record our knowledge of Jane's employment with State University. Instead of using now as the "to" time in Jane's tuple, we can use an indeterminate instant. Which indeterminate instant to use depends on our knowledge of the situation. If Jane was hired to work at least two months, we could record this information as shown in Figure 6(a). Here two time bounds, July 31 and forever, delimit the "to" indeterminate instant. If State University has a mandatory retirement policy, we could decrease the indeterminacy considerably, as shown in Figure 6(b).

Indeterminate instants address the pessimistic update assumption, providing evidence that Jane might still be employed in the future. They also remove the problem of incompleteness in the nontimestamp attributes [e.g., possibly employed, as shown in Figure 1(c)], and ensure that new knowledge acquired later, such as the information that Jane left the company on August 10, is not inconsistent with currently stored information, but rather is a refinement of that information. They also address the problem of now in predictive updates; an indeterminate interval is a valid interval no matter when it was stored in the database.

There are two bounds on the information represented by an indeterminate interval [Lipski 1979]. The first bound is the definite information. The 
definite information represents all that is definitely known about the interval and is the intersection of all the possible intervals. The second bound is the possible information. The possible information represents the maximum possible extent of an interval and is the union of all the possible intervals. The two bounds have different extensionalizations. The definite information is given by the definite extensionalization, presented next.

Definition 4.7 [Indeterminate Ground Tuple]. An indeterminate ground tuple is a ground tuple of the form $T=\left\langle X\right.$, $\left.\left[v t_{1} \sim v t_{2}, v t_{3} \sim v t_{4}\right]\right\rangle$, where $v t_{1} \leq v t_{2}$ and $v t_{3} \leq v t_{4}$. Here, $v t_{1}$ and $v t_{2}$ are the lower and upper bound, respectively, of the starting instant and $v t_{3}$ and $v t_{4}$ are the lower and upper bound, respectively, of the terminating instant.

Definition 4.8 [Definite Extensionalization of an Indeterminate Tuple]. The definite extensionalization of an indeterminate ground tuple of the form $T=\left\langle X,\left[v t_{1} \sim v t_{2}, v t_{3} \sim v t_{4}\right]\right\rangle$, at the reference time $r t_{*}$ is defined as follows.

$$
\llbracket T \rrbracket_{r t^{*}}^{D}={ }_{d f}\left\{\left(X, v t, r t_{*}\right) \mid v t \in\left[\left\langle\left\langle v t_{2}\right\rangle\right\rangle_{r t *},\left\langle\left\langle v t_{3}\right\rangle\right\rangle_{r t *}\right]\right\} .
$$

The possible information is given by the possible extensionalization.

Definition 4.9 [Possible Extensionalization of an Indeterminate Tuple]. The possible extensionalization of a ground indeterminate tuple of the form $T=\left\langle X,\left[v t_{1} \sim v t_{2}, v t_{3} \sim v t_{4}\right]\right\rangle$ at the reference time $r t_{*}$ is defined as follows.

$$
\llbracket T \rrbracket_{r t *}^{P}={ }_{d f}\left\{\left(X, v t, r t_{*}\right) \mid v t \in\left[\left\langle\left\langle v t_{1}\right\rangle\right\rangle_{r t *},\left\langle\left\langle v t_{4}\right\rangle\right\rangle_{r t *}\right]\right\} .
$$

It is always the case that the definite information is a subset of the possible information. Note that if the bounding instants are determinate, that is, if the lower and upper bounds are the same, then the possible and definite extensionalizations yield exactly the same set of tuples. Consequently, for the extensionalization of determinate intervals, we omit the possible or definite superscript and use $\llbracket \mathbb{1}_{r t_{*}}$ instead of either $\mathbb{\|} \mathbb{1}_{r t_{*}}^{P}$ or $\mathbb{\|} \mathbb{}_{r t_{*}}^{D}$.

Valid-time tuples timestamped with indeterminate intervals have a graphical representation similar to the one described. Both the possible and definite extensionalizations are represented. We use different shadings to distinguish the regions in the two extensionalizations. As an example, assume that the academic career of Jane at State University is given by the tuple

\section{$\langle$ Jane, Assistant, [June $1 \sim$ June 3, June $7 \sim$ June 10]〉.}

Jane's academic career, for the reference times [June 1, June 11], is graphically represented in Figure 7 (a). Note that the region of possible information is never smaller than the region of definite information and that the valid time is reference-time invariant (just as it is for determinate intervals) when the tuple has no variables. 


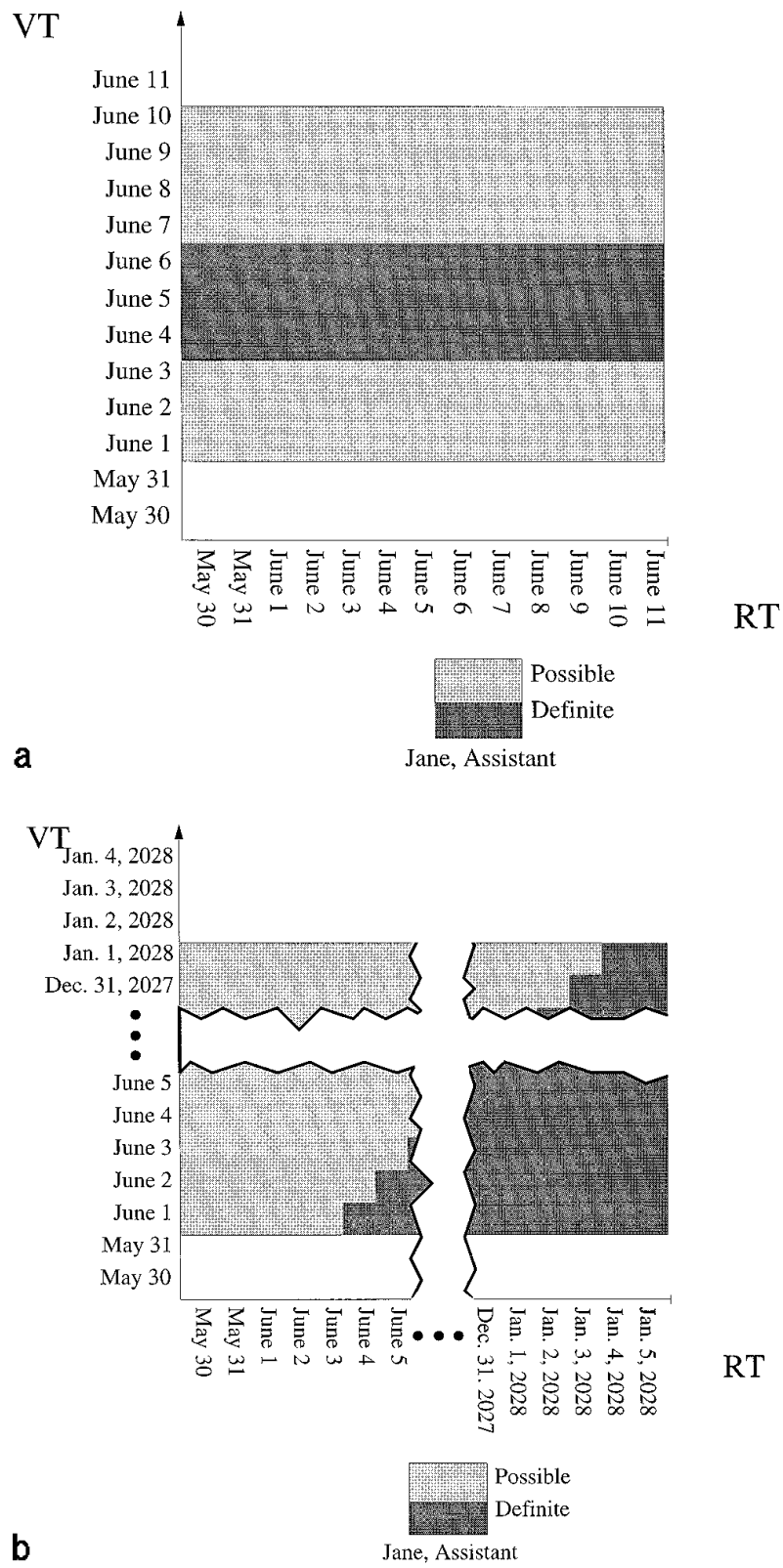

Fig. 7. Graphical representation of extensionalization of an indeterminate valid-time tuple: (a) ground indeterminate valid-time tuple; (b) Jane's possible and definite employment.

\subsection{Now-Relative Indeterminate Instants}

To achieve the full benefit of indeterminate timestamp values, we proceed by introducing now-relative indeterminate instants, which may be understood as generalizations of the ground indeterminate timestamps and of the now-relative instants presented earlier. 


\begin{tabular}{|r|r||r|r|}
\hline \multicolumn{3}{|c|}{ FACULTY } \\
\hline NAME & RANK & (from) & VALID TIME \\
(to)
\end{tabular}

(a)

\begin{tabular}{|l|l||l|l|}
\hline Jane & Assistant & June 1 & July $6 \sim$ January 1, 2028 \\
\hline
\end{tabular}

(b)

\begin{tabular}{|l|c||c|c|}
\hline Jane & Assistant & June 1 & July 7 $~$ January 1, 2028 \\
\hline
\end{tabular}

(c) \begin{tabular}{|l|l||l|l|}
\hline Jane & Assistant & June 1 & now -3 days $\sim$ January 1, 2028 \\
\hline
\end{tabular}

Fig. 8. Using indeterminate and now-relative indeterminate timestamps.

To exemplify and motivate the utility of this new type of instant, assume that today is July 9, that Jane is still employed, and that there is at most a three-day lag in recording a fact in the database. Jane's tuple in the database should not be that of Figure 6(b), but rather that shown in Figure 8(a) which is more accurate. The state on July 10 is shown in Figure 8(b). Note how the indeterminacy in the "to" instant has decreased ever so slightly_on July 10 we know that Jane was employed on July 7.

To accurately represent our continuously changing knowledge about Jane's employment, we need to combine now-relative instants and ground indeterminate values into a new kind of instant, which we call a nowrelative indeterminate instant. An example is shown in Figure 8(c) where the "to" timestamp is such an instant. Note that a tuple with a now-relative indeterminate instant may yield no definite information or may have the same possible and definite information content; it all depends upon when we observe that tuple.

The visualization of a tuple at the extensional database level with a now-relative indeterminate time is similar to the visualization of a tuple with an indeterminate interval. Both the definite and possible regions are plotted on the same graph but using different colors or patterns. Figure 7(b) shows a graph of both the possible and definite extensionalizations of the tuple in Figure 8(c) for every reference time between May 30 and January 5, 2028. Note that for all reference times before June 4 the tuple does not contain any definite information, only possible information. The definite information gradually increases as the reference time advances. On January 4, 2028, and for all reference times thereafter, the possible and definite information for the tuple are the same.

Now-relative indeterminate instants provide a flexible means of precisely capturing our imprecise, but current-time dependent, knowledge of when a fact is valid. For instance, in the tuple given in Figure 8(c), we are certain that Jane was an Assistant Professor starting on June 1, but our knowledge of when she ceases to be an Assistant Professor is imprecise; all we know is that she was definitely an Assistant Professor until three days ago and that it is possible that she will remain an Assistant Professor until retirement on January 1, 2028. The "to" timestamp allows us to capture this precisely. Using a now-relative indeterminate instant ensures that continual updates are not required, while capturing all of our knowledge of exactly when Jane is employed by State University. 
Table I. Extensionalization of Valid-Time Databases

\begin{tabular}{|l|l|l|}
\hline & Variable Database & Extensional Database \\
\hline $\mathbf{v} 1$ & $T=<X,\left[v t_{1}, v t_{2}\right]>$ & $\llbracket T \rrbracket_{r t_{*}}=\left\{\left(X, v t, r t_{*}\right) \mid v t_{1} \leq v t \leq v t_{2}\right\}$ \\
\hline $\mathbf{v} 2$ & $T=<X,\left[v t_{1}, n o w \pm n\right.$ days $]>$ & $\llbracket T \rrbracket_{r t_{*}}=\left\{\left(X, v t, r t_{*}\right) \mid v t_{1} \leq v t \leq r t_{*} \pm n\right\}$ \\
\hline $\mathbf{v 3}^{D}$ & $T=<X,\left[v t_{1} \sim v t_{2}, v t_{3} \sim v t_{4}\right]>$ & $\llbracket T \rrbracket_{r t_{*}}^{D}=\left\{\left(X, v t, r t_{*}\right) \mid v t_{2} \leq v t \leq v t_{3}\right\}$ \\
$\mathbf{v 3}^{P}$ & $T=<X,\left[v t_{1} \sim v t_{2}, v t_{3} \sim v t_{4}\right]>$ & $\llbracket T \rrbracket_{r t_{*}}^{P}=\left\{\left(X, v t, r t_{*}\right) \mid v t_{1} \leq v t \leq v t_{4}\right\}$ \\
\hline $\mathbf{v 4}^{D}$ & $T=<X,\left[v t_{1}, n o w \pm n\right.$ days $\left.\sim v t_{2}\right]>$ & $\llbracket T \rrbracket_{r t_{*}}^{D}=\left\{\left(X, v t, r t_{*}\right) \mid v t_{1} \leq v t\right.$ \\
$\mathbf{v 4}^{P}$ & $T=<X,\left[v t_{1}, n o w \pm n\right.$ days $\left.\sim v t_{2}\right]>$ & $\llbracket T \prod_{r t_{*}}^{P}=\left\{\left(X, v t, r t_{*}\right) \mid v t_{1} \leq v t \leq v t_{2}\right\}$ \\
\hline
\end{tabular}

A now-relative indeterminate instant consists of a variable lower bound and a ground upper bound. The lower bound cannot exceed the instant's upper bound; consequently the upper bound represents a limit on the possible or definite information in the instant. So, for instance, the possible or definite information represented by Jane's employment tuple shown in Figure 8(c) cannot extend beyond January 1, 2028, even if today is after January 1, 2028. If today is May 9, then the lower bound is May 6 and the tuple indicates that we expect Jane to be (possibly) employed from June 1 to January 1, 2028. If today is January 1, 2050, then the upper bound is January 1, 2028 and the tuple indicates that Jane was actually employed from June 1 to January 1, 2028. In short, now-relative indeterminate instants capture the semantics of predictive updates. They are also able to model the evolutionary character of temporal databases since values in the possible extensionalization of a tuple evolve into definite values as the reference time increases.

Definition 4.10 [Possible Extensionalization of a Now-Relative Indeterminate Tuple]. The possible extensionalization at reference time $r t_{*}$ of the tuple $T=\left\langle X,\left[e_{1} \sim v t_{2}, e_{3} \sim v t_{4}\right]\right.$, $\rangle$ where $e_{1}$ and $e_{3}$ stand for "expressions using variables," and $v t_{2}$ and $v t_{4}$ are ground values, is defined as follows.

$$
\llbracket T \rrbracket_{r t^{*}}^{P}={ }_{d f}\left\{\left(X, v t, r t_{*}\right) \mid v t \in\left[\min \left(\left\langle\left\langle e_{1}\right\rangle\right\rangle_{r t^{*}},\left\langle\left\langle v t_{2}\right\rangle\right\rangle_{r t_{*}}\right),\left\langle\left\langle v t_{4}\right\rangle\right\rangle_{r t_{*}}\right]\right\} .
$$

Definition 4.11 [Definite Extensionalization of a Now-Relative Indeterminate Tuple]. The definite extensionalization of the tuple $T=\left\langle X,\left[e_{1} \sim v t_{2}\right.\right.$, $\left.\left.e_{3} \sim v t_{4}\right],\right\rangle$ at reference time $r t_{*}$ is defined as follows.

$$
\llbracket T \rrbracket_{r t^{*}}^{D}={ }_{d f}\left\{\left(X, v t, r t_{*}\right) \mid v t \in\left[\left\langle\left\langle v t_{2}\right\rangle\right\rangle_{r t^{*}}, \min \left(\left\langle\left\langle e_{3}\right\rangle\right\rangle_{r t^{*}},\left\langle\left\langle v t_{4}\right\rangle\right\rangle_{r t^{*}}\right)\right]\right\} .
$$

\subsection{Summary of Extensionalizations}

Table I summarizes some of the valid-time extensionalizations (the most representative cases). Case $\mathbf{v 1}$ (the $\mathbf{v}$ stands for "valid-time" database) specifies the extensionalization of tuple timestamped with a determinate interval, case v2 a now-relative interval, case v3 an indeterminate interval, and case $\mathbf{v} 4$ a now-relative indeterminate interval. Note that the possible and definite extensionalizations in cases $\mathbf{v} \mathbf{1}$ and $\mathbf{v} \mathbf{2}$ are the same since the intervals are determinate. 


\subsection{Querying Variable Valid-Time Databases}

In this section we enhance the query facilities of existing (nonvariable) data models to support queries on timestamps containing variables. The essential problem is what to do when a variable is encountered during query evaluation. In the following we describe a solution to that problem. Further, we show how the framework may be utilized in defining algebraic operators on variable databases that are consistent with the semantics of variable databases. Specifically, we consider the valid-time timeslice operation.

When evaluating a user-level query, for example, written in some dialect of SQL, it is common to transform it into an internal algebraic form that is suitable for subsequent rule or cost-based query optimization. As the query processor and optimizer are among the most complex components of a database management system, it is important that the added functionality of current-time-related timestamps necessitates only minimal changes to these components.

Although many solutions may be envisioned, a solution that meets this requirement and is natural in our semantic framework is to eliminate variables before they are seen. More specifically, when a timestamp that contains a variable is used during query processing (e.g., in a test for overlap with another timestamp), a ground version of that timestamp is created and is used instead. Thus, only minimal, incremental changes to the query processor are needed. Existing components remain unchanged. Only a new component that substitutes variable timestamps with ground timestamps has to be added.

More specifically, we define a "bind" operator that is added to the set of operators already present. When user-level queries are mapped to the internal representation, this operator is utilized. The operator accepts any valid-time tuple with variables as defined earlier in the article. It substitutes a ground value for each variable and thus returns a ground (but still variable-level) tuple.

To exemplify, assume that on June 20 we are interested in Jane's employment status at State University as of June 15 and that we have available the database with the single tuple in Figure 1(a), but that our query processor is unable to contend with variables in timestamps. To answer the query, we first eliminate the variable now by applying the bind operator (defined in the following) to the tuple, resulting in $\{\langle$ Jane, Assistant, [June 1, June 20] $\rangle$. Second, this tuple is passed to the query processor, where it is then used to compute that Jane is an Assistant Professor on June 15.

Definition 4.12 [Variable-Level Valid-Time Bind]. Given an arbitrary valid-time tuple $T=\left\langle X,\left[e_{1} \sim v t_{2}, e_{3} \sim v t_{4}\right]\right\rangle$ and a reference time $r t_{*}$, the variable-level valid-time bind operation eliminates all variables and is defined as follows.

$$
\operatorname{bind}_{r t_{*}}^{V, V T}(T)=_{d f}\left\langle X,\left[\left\langle\left\langle e_{1}\right\rangle\right\rangle_{r t_{*}} \sim\left\langle\left\langle v t_{2}\right\rangle\right\rangle_{r t *},\left\langle\left\langle e_{3}\right\rangle\right\rangle_{r t_{*}} \sim\left\langle\left\langle v t_{4}\right\rangle\right\rangle_{r t *}\right]\right\rangle .
$$


This operation can be extended in the obvious way to an operator on sets of tuples (i.e., relations). The superscript " $V, V T$ " indicates that this is a variable-level, valid-time operator. Note that two tuples that have timestamps

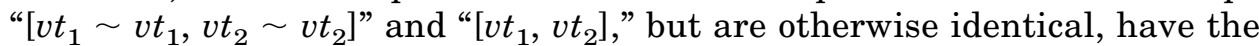
same extensionalizations. Thus the timestamps are equivalent, and therefore the preceding definition also covers determinate timestamps.

The outcome of a query on a variable database generally depends on the specific reference-time argument given to the bind operator. To provide a foundation for understanding how to use the bind operator when mapping user-level queries to algebraic equivalents, we must explore its meaning.

The bind operator with reference-time argument $r t *$ replaces each variable by its denotation or value at time $r t_{*}$. Put differently, the operator replaces each variable timestamp with a ground timestamp that has the special property of having the same denotation, or value, as the variable timestamp at the reference time $r t_{*}$. At other reference times, the original and the ground timestamps will generally not have the same denotation. This semantics may be expressed at the extensional level as follows.

Definition 4.13 [Extensional-Level Valid-Time Bind]. Given an arbitrary set $S$ of extensional-level valid-time tuples of the form $(X, v t, r t)$ and a reference time $r t_{*}$, the extensional-level valid-time bind operation is defined as follows.

$$
\operatorname{bind}_{r t_{*}}^{E, V T}(S)={ }_{d f}\left\{(X, v t, r t) \mid\left(X, v t, r t_{*}\right) \in S \vee r t \in \mathscr{T}_{R T}\right\}
$$

The " $E$ " in the operator's superscript indicates that this is an extensionallevel operator. At the extensional level, the bind operator chooses the meaning of a tuple at the indicated reference time and propagates that meaning over every possible reference time, resulting in a reference-time invariant meaning. To prove that this definition is correct vis-á-vis the required commutativity of the left side of the diagram in Figure 4, we need to show that given an arbitrary tuple $T$, and a reference time $r t_{*}$, $\llbracket \operatorname{bind}_{r t_{*}}^{V, V T}(T) \rrbracket=$ bind $_{r t_{*}}^{E, V T}(\llbracket T \rrbracket)$. This follows directly from the definitions. For brevity, we omit the proof.

Intuitively, the bind operator sets the perspective of the observer (i.e., it sets the reference time as described in Section 3.2). Existing query languages generally assume that the perspective of a user observing the database is the same as what we termed the query time or current time and denoted $t_{\text {current }}$ in that section. However, as we will see, a bind operator provides a basis for added functionality.

Recall that the definition of query operators at the variable level is complex and that current temporal data models have not satisfactorily resolved the complex problems involved. In our approach, we first preprocess the variable-level database by binding timestamps to $r t_{*}$, effectively removing the variables. We can then apply any algebraic operators from an existing temporal query language. It should be clear from the previous discussion that the composition of bind with any of these algebraic operators is well-defined, and the timestamps have the appropriate meaning. 
To show how operators are defined within the semantic framework, we now define several timeslice operators. Valid-time timeslice is a fairly standard operation; some variant of timeslice is a component of virtually all temporal algebras. Standard definitions of determinate and indeterminate timeslice operators are given in the following. Note that these do not have to contend with variables; because of the use of the bind operator, they can be defined solely on ground tuples.

Definition 4.14 [Variable-Level Definite Valid-Time Timeslice]. Let $S$ be a set of tuples at the variable database level, that is, a set of tuples of the form $T=\left\langle X,\left[v t_{1} \sim v t_{2}, v t_{3} \sim v t_{4}\right]\right\rangle$, where the $v t_{i}$ are ground values. The definite valid-time timeslice of $S$ at valid time $v t_{*}$ is defined as follows.

$\prod_{v t *}^{D, V, V T}$

$=_{d f}\left\{\left\langle X,\left[v t_{*}, v t_{*}\right]\right\rangle \mid T=\left\langle X,\left[v t_{1} \sim v t_{2}, v t_{3} \sim v t_{4}\right]\right\rangle \in S\left(v t_{*} \in\left[v t_{2}, v t_{3}\right]\right)\right\}$.

Definition 4.15 [Variable-Level Possible Valid-Time Timeslice]. Let $S$ be a set of tuples at the variable database level, that is, a set of tuples of the form $T=\left\langle X,\left[v t_{1} \sim v t_{2}, v t_{3} \sim v t_{4}\right]\right\rangle$, where the $v t_{i}$ are ground values. The possible valid-time timeslice of $S$ at valid time $v t_{*}$ is defined as follows.

$$
\begin{aligned}
& \prod_{v t^{*}}^{P, V T}(S) \\
& \quad={ }_{d f}\left\{\left\langle X,\left[v t_{*}, v t_{*}\right]\right\rangle \mid \quad T=\left\langle X,\left[v t_{1} \sim v t_{2}, v t_{3} \sim v t_{4}\right]\right\rangle \in S\left(v t_{*} \in\left[v t_{1}, v t_{4}\right]\right)\right\} .
\end{aligned}
$$

The superscript " $D, V, V T$ " of the first operator indicates that it considers only the definite information content, that it belongs at the variable level, and that it is a valid-time timeslice. Also, recall that definite timestamps are special cases of indeterminate timestamps, which are then also covered by the definition. The straightforward extensions of the operator to slice on valid-time intervals and to take as input a set of tuples (i.e., a relation) are omitted for brevity. A timeslice operator at the extensional level that satisfies the correctness criterion of the framework, as illustrated in Figure 3, specifically, $\Pi_{v t_{*}}^{E, V t}\left(\llbracket T \rrbracket^{D}\right)=\llbracket \Pi_{v t_{*}}^{D, V, V T}(T) \rrbracket$, is given next. The proof of this statement is omitted for space considerations.

Definition 4.16 [Extensional-Level Valid-Time Timeslice]. Since there is no indeterminacy at the extensional database level, there is no need for two timeslice operators; one suffices. At the extensional database level, the valid-time timeslice of a set $S$ consisting of tuples of the form $(X, v t, r t)$ is defined as follows.

$$
\prod_{v t^{*}}^{E, V T}(S)={ }_{d f}\left\{\left(X, v t_{*}, r t\right) \mid\left(X, v t_{*}, r t\right) \in S\right\} .
$$

We are now in a position to explore the interaction of the important times (Section 3.2) by using the new bind operator and existing (variable-level) timeslice operators. We generally consider only the definite version of the timeslice operator. 
The bind operator sets the perspective and is combined with timeslice to formulate queries. In the first two example queries given in the following, we assume that the database is to be observed from the perspective of June 5 (i.e., $r t_{*}=$ June 5 ). For all examples, the query time is assumed to also be June 5 (i.e., $t_{\text {current }}=$ June 5 ).

-Who is employed on June 5 ?

$$
\prod_{\text {June } 5}^{D, V, V T}\left(\text { bind }_{\text {June } 5}^{V, V T}(\text { Faculty) })\right. \text {. }
$$

—Who will actually be employed on June 7 ?

$$
\prod_{\text {June } 7}^{D, V, V T}\left(\text { bind }_{\text {June } 5}^{V, V T}(\text { Faculty) })\right. \text {. }
$$

Tuples with a "to" time of now will not be in the result.

-Discounting future (and as yet unknown) employee hirings or firings, who do we expect to be employed on June 7 ?

$$
\prod_{\text {June } 7}^{D, V, V T}\left(\text { bind }_{\text {June }}^{V, V T}(\text { Faculty) })\right. \text {. }
$$

Our "expectation" is that current employees (i.e., those employed now) will remain employed through June 7 . We make this expectation concrete by adopting a June 7 perspective of the database. Then all tuples with a "to" time of now will contribute to the result.

-Making no assumptions about the future evolution of the database, who will possibly be employed on June 7 ?

$$
\prod_{\text {June } 7}^{P, V, V T}\left(\text { bind }_{\text {June } 5}^{V, V T}(\text { Faculty) })\right. \text {. }
$$

We limit the future evolution of the database by adopting a June 5 perspective, and query about a possible future from that perspective. Tuples with intervals with a "to" time of now June 7 (or a later upper bound) will be in the result, although those with a "to" time of now will not be in the result.

We have seen that the binding of now has an impact on the meaning of query results and that query results must be interpreted with respect to a particular perspective. Existing query languages (e.g., TSQL2 [Snodgrass 1995]) generally assume that the perspective and the query time coincide. This assumption leads to a restriction in functionality, but it also simplifies the interpretation of answers.

\subsection{Summary}

Now appears in many temporal database models, although it is sometimes disguised under a different name. Now is commonly used as the "to" time in a valid-time tuple. It has one principal advantage: it efficiently represents that a tuple will continue to be valid, barring further updates. But it also suffers from several anomalies, as discussed in Section 2.2. In the follow- 
ing, we show how each of these four anomalies is addressed in our approach.

The use of now as a "to" time makes a pessimistic assumption about a tuple's continuing validity since it indicates that a tuple's validity ends immediately, whereas we expect such tuples to remain valid in future. To address this problem we propose a semantics that allows users to bind now to any desired "perspective," that is, any reference time, in a query. The user can adopt a pessimistic perspective, by binding now to the current time, or an optimistic perspective, by binding now to some future time, for example, forever. The proposed semantics is backwards-compatible with existing, nonvariable semantics.

Now also imposes an unrealistic assumption about the punctuality of updates to a tuple because it presupposes that the current database state accurately models the current real-world state. To address this anomaly we introduce now-relative instants that include a displacement from now. Now-relative instants can relax the strict punctuality assumption by using the displacement from now to model the real-world delay in updating tuples.

Further, the use of now as a timestamp value necessitates special-case processing to correctly support predictive updates. A predictive update inserts into a database a fact that is valid sometime in the future. If such a fact has a "to" time of now, its valid time ends before it starts. This not only violates a common assumption about interval timestamps (that the timestamp is a valid interval), it can also lead to an incorrect result for a query about information valid in the future. To support predictive update we propose now-relative indeterminate instants that combine indeterminacy with now-relativity. An interval with a now-relative indeterminate instant as the "to" time is a valid interval no matter when it is inserted into the database. Furthermore, the indeterminacy in a now-relative indeterminate instant can be used to model the uncertainty of future information, whereas the now-relative portion of the instant relaxes the punctuality assumption and allows the user to adopt both optimistic and pessimistic query perspectives.

Finally, when querying data that involves now, the current time must be clearly specified since the value of now depends on this time. An unclear specification can result in ambiguous query results. In our proposed framework, the current time is fixed by the bind operation. This allows the perspective of the observer to be set, thereby ensuring that the same answer is always returned for a particular reference time.

\section{TRANSACTION-TIME DATABASES}

The use of a current-time variable in the transaction-time dimension is not as fraught with problems as its use in the valid-time dimension. The reason for this lies in the different meaning of transaction time in a database. The valid time of a tuple indicates when it is considered valid, and, as such, valid timestamps of tuples are generally provided by the users. In contrast, 
transaction timestamps are supplied by the database management system itself. This is a consequence of the meaning of transaction time: the transaction timestamp indicates when the tuple is current in the database.

Although several timestamp values (e.g., forever and now) have been used, it is our contention that they all have the same meaning. Specifically, they are all employed as a "stop" timestamp that indicates that the tuple stamped is current (from the "start" time) until the database is updated to indicate otherwise. However, the various names used do not convey the intuitive semantics of the variable in this dimension. A term more precise than now or forever for this meaning of "not yet logically deleted or updated" is until changed - a fact is current in the database until changed. It has no counterpart in valid time. Using until changed instead of now avoids also potential confusion with now in valid time, although some authors have used until changed in valid time [Wiederhold et al. 1993]. Unlike the (valid-time variable) now, until changed can only be used as the "stop" time; it is undefined to use it as the "start" time.

\subsection{Extensionalization of a Ground Transaction-Time Tuple}

We first examine the meaning of a tuple without variables in transaction time. The extensionalization of such a tuple differs from its valid-time counterpart, because the semantics of transaction time does not allow future transaction times to be recorded in the database. Hence, the extensionalization of such tuples must be restricted to ensure that no matter when we look at the database, we can never see a "future" transaction time. Since the future depends on when we observe the database, the reference time is used to constrain the transaction-time in the expanded set of tuples.

In Definition 4.1, the denotation at any reference time of a ground valid-time instant was given to be the instant itself. The same applies to ground transaction-time instants.

Definition 5.1 [Transaction-Time Extensionalization of a Ground Tuple]. The transaction-time extensionalization of a tuple of the form $T=\left\langle X,\left[t t_{1}\right.\right.$, $\left.\left.t t_{2}\right]\right\rangle$, where $X$ is some set of attribute values and $t t_{1}$ and $t t_{2}$ are transactiontime instants, at the reference time $r t_{*}$, where $t_{0} \leq r t_{*} \leq t_{\text {current }}$, is defined as follows.

$$
\llbracket T \rrbracket_{r t *}^{T T}={ }_{d f}\left\{\left(X, t t, r t_{*}\right) \mid t t \in\left[\left\langle\left\langle t t_{1}\right\rangle\right\rangle_{r t *}, \min \left(\left\langle\left\langle t t_{2}\right\rangle\right\rangle_{r t *}, r t_{*}\right)\right]\right\} .
$$

We use a "TT" superscript to differentiate this mapping from a valid-time extensionalization.

The visualization of a transaction-time tuple is similar to that of a valid-time tuple. Again, a two-dimensional graph is used. The $X$-axis of the graph is the reference time, and the $Y$-axis is the transaction time. However, unlike a valid-time tuple without variables, the transaction-time interval for a tuple is not independent of the time at which we observe the tuple. Figure 9 depicts the extensionalization of the transaction-time tuple 〈Jane, Assistant, [June 5, June 8] 〉 for a sequence of reference times, June 1 


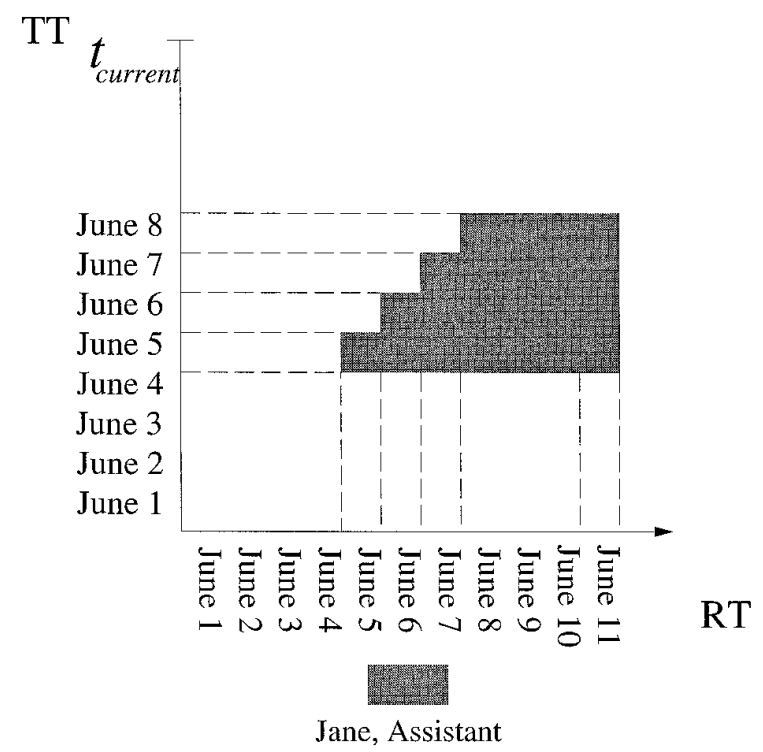

Fig. 9. Graphical representation of transaction-time tuple.

through June 11. Note that the depicted region has a "stair-shaped" feature which is a result of the constraint that the transaction time cannot exceed the reference time.

\subsection{Semantics of "Until Changed"}

The current-time variable in transaction time indicates that the associated fact is current in the database until the fact is changed by a subsequent update. Substituting transaction time for valid time in our running example yields the relation shown in Figure 10.

Definition 5.2 [Denotation of Until Changed]. The denotation of the transaction-time variable until changed at a particular reference time $r t_{*}$, where $t_{0} \leq r t_{*} \leq t_{\text {current }}$, is defined as follows.

$$
\langle\langle\text { until changed }\rangle\rangle_{r t *}={ }_{d f} r t_{*} .
$$

The extensionalization of a transaction-time tuple with the variable until changed as the value of its "stop" time is obtained by generating tuples for each instant in the ground interval that results from substituting until changed by $r t_{*}$. Thus, Definition 5.1 also applies when until changed is allowed as a "stop" time.

\subsection{Summary of Extensionalizations}

Table II summarizes the extensionalizations presented for transaction time. Case t1 (the t stands for "transaction-time" database) applies to tuples with fully ground timestamp values only, whereas Case $\mathbf{t} 2$ covers the case where until changed is the "stop" time. 


\begin{tabular}{|l|c||c|c|}
\hline \multicolumn{4}{|c|}{ FACULTY } \\
\hline \multirow{2}{*}{ NAME } & \multicolumn{1}{|c||}{ RANK } & \multicolumn{2}{|c|}{ TRANS TIME } \\
(start) & (stop) \\
\hline \hline Jane & Assistant & June 1 & until changed \\
\hline
\end{tabular}

Fig. 10. Using until changed in a transaction-time relation.

Table II. Extensionalization of Transaction-Time Databases

\begin{tabular}{|l|l|l|}
\hline & Variable Database & Extensional Database \\
\hline $\mathbf{t 1}$ & $T=<X,\left[t t_{1}, t t_{2}\right]>$ & $\llbracket T \rrbracket_{r t_{*}}=\left\{\left(X, t t, r t_{*}\right) \mid t t_{1} \leq t t \leq \min \left(t t_{2}, r t_{*}\right)\right\}$ \\
$\mathbf{t 2}$ & $T=<X,\left[t t_{1}\right.$, until changed $]>$ & $\llbracket T \rrbracket_{r t_{*}}=\left\{\left(X, t t, r t_{*}\right) \mid t t_{1} \leq t t \leq r t_{*}\right\}$ \\
\hline
\end{tabular}

\subsection{Querying Variable Transaction-Time Databases}

The bind operator for transaction time eliminates occurrences of until changed in the "stop" component of timestamps.

Definition 5.3 [Variable-Level Transaction-Time Bind]. Given a tuple $T=\left\langle X,\left[t t_{1}, e_{2}\right]\right\rangle$, where $t t_{1}$ is a ground transaction time and $e_{2}$ is until changed or a ground transaction time, the variable-level transaction-time bind operation is defined as follows.

$$
\operatorname{bind}^{V, T T}(T)=_{d f}\left\langle X,\left[\left\langle\left\langle t t_{1}\right\rangle\right\rangle_{t_{\text {current }}},\left\langle\left\langle e_{2}\right\rangle\right\rangle_{t_{\text {current }}}\right]\right\rangle .
$$

Again, this operation can be extended in the obvious way to relations. Transaction-time bind is very similar to valid-time bind, but differs in one important respect. The bind ${ }^{V, T T}$ operator does not accept any time argument, but always binds until changed to the query time or current transaction time, $t_{\text {current }}$.

Since the bind ${ }^{V, T T}$ operator lacks a time parameter and is always applied before any other operator, it is feasible to omit the operator and instead build it into the transaction timeslice operator, as has been done in some variable-level transaction-time algebras [Jensen and Mark 1992]. However, it would also need to be built into any additional operators, so to preserve the parallel with Section 4.6, we choose not to do this. The definition of the extensional-level bind for transaction time is omitted because it is very similar to Definition 4.13.

Definition 5.4 [Variable-Level Transaction-Time Timeslice]. Let $S$ be a set of tuples at the variable database level, that, a set of tuples of the form $T=\left\langle X,\left[t t_{1}, t t_{2}\right]\right\rangle$, where $t t_{1}$ and $t t_{2}$ are ground transaction times. The transaction-time timeslice of $S$ at transaction-time $t t_{*}$ is defined as follows.

$$
\prod_{t t *}^{V, T T}(S)={ }_{d f}\left\{\left\langle X,\left[t t_{*}, t t_{*}\right]\right\rangle \mid \quad T=\left\langle X,\left[t t_{1}, t t_{2}\right]\right\rangle \in S\left(t t_{*} \in\left[t t_{1}, t t_{2}\right]\right)\right\} .
$$

Definition 5.5 [Extensional-Level Transaction-Time Timeslice]. At the extensional database level, the transaction-time timeslice of a set $S$ consisting of tuples of the form $(X, t t, r t)$ is defined as follows.

$$
\prod_{t t_{*}}^{E, T T}(S)=_{d f}\left\{\left(X, t t_{*}, r t\right) \mid\left(X, t t_{*}, r t\right) \in S\right\} .
$$


The definitions of transaction-time binding and slicing conform to the framework we set up in Section 3.3, specifically to Figure 4; that is, $\Pi_{t t_{*}}^{E, T T}\left(\right.$ bind $_{r t_{*}}^{E, T T}\left(\llbracket S \rrbracket_{r t_{*}}^{T T}\right)=\llbracket \Pi_{t t_{*}}^{V, T T}\left(\operatorname{bind}_{r t_{*}}^{V, T T}(S)\right) \rrbracket_{r t_{*}}^{T T}$. The proof, which follows from the definitions, is omitted for brevity.

As with valid-time queries, a combination of bind and timeslice supports transaction-time queries. When querying about a transaction-time database, there are some important times to consider: (i) the transaction-time timeslice time $t t_{*}$, indicating that information is sought that was current in the database at time $t t_{*}$, and (ii) the query time $t_{\text {current }}$, the time at which the query is asked.

As an example, we consider several timeslice operations on the tuple $T$ depicted in Figure 2(a). For the following queries, it is assumed that $t_{\text {current }}$ is June 11.

- $\prod_{\text {June }}^{V, T T}\left(\right.$ bind $\left.^{V, T T}(T)\right)$ yields an empty result because the interval associated with $T$ is before the timeslice time-tuple $T$ ceased to be current starting on June 9.

- $\Pi_{\text {June }}^{V, T T}\left(\right.$ bind $\left.^{V, T T}(T)\right)$ yields tuple $T$, but with "start" and "stop" times of June 7. This is so because the information recorded by $T$ was current on June 7.

\section{BITEMPORAL DATABASES}

A bitemporal relation supports both transaction and valid time [Jensen et al. 1994a; Snodgrass and Ahn 1985]. The combination of these two temporal dimensions empowers the database to record time-dependent information as well as earlier database states. Bitemporal databases thus combine the advantages of valid-time and transaction-time databases. Yet this greater flexibility comes at a cost: increased complexity derives from the interactions between the two temporal dimensions which must be carefully considered. The logical framework we have presented for current-time variables has been designed to make it relatively straightforward to obtain the semantics of bitemporal databases. The interaction between the current-time variable for valid time, now, and transaction time, until changed, is coordinated through the reference time. We demonstrate one possible (and, we think, reasonable) semantics for this combination, but we emphasize that the framework is general enough to allow the definition of other alternative semantics for the interaction of these variables.

\subsection{Extensionalization of Bitemporal Databases}

The timestamp of a bitemporal tuple contains both a valid-time and a transaction-time component. Since the valid-time component may be indeterminate, it is necessary to distinguish between a definite and a possible extensionalization, $\llbracket \rrbracket_{r t_{*}}^{B T, D}$ and $\llbracket \rrbracket_{r t_{*}}^{B T, P}$, respectively.

Definition 6.1 [Definite Extensionalization of a Bitemporal Tuple]. The definite extensionalization of a bitemporal tuple $T$ of the form $T=\langle X$, $\left.\left[v t_{1}, v t_{2}\right],\left[t t_{1}, t t_{2}\right]\right\rangle$, where $X$ is some set of attribute values and the 
timestamp $\left[v t_{1}, v t_{2}\right],\left[t t_{1}, t t_{2}\right]$ may contain any of the variables introduced earlier, at the reference time $r t_{*}$ is defined as follows.

$$
\begin{aligned}
\llbracket T \rrbracket_{r t *}^{B T, D=}=_{d f}\left\{\left(X, v t, t t, r t_{*}\right) \mid\left(X, v t, r t_{*}\right)\right. & \in \llbracket\left\langle X,\left[v t_{1}, v t_{2}\right]\right\rangle \rrbracket_{r t *}^{V T, D} \\
& \left.\wedge\left(X, t t, r t_{*}\right) \in \llbracket\left\langle X,\left[t t_{1}, t t_{2}\right]\right\rangle \rrbracket_{r t *}^{T T}\right\} .
\end{aligned}
$$

Definition 6.2 [Possible Extensionalization of a Bitemporal Tuple]. The possible extensionalization of a bitemporal tuple $T$ of the form $T=\left\langle X,\left[v t_{1}, v t_{2}\right],\left[t t_{1}, t t_{2}\right]\right\rangle$ at the reference time $r t_{*}$ is defined as follows.

$$
\begin{aligned}
\llbracket T \rrbracket_{r t *}^{B T, P}={ }_{d f}\left\{\left(X, v t, t t, r t_{*}\right) \mid\left(X, v t, r t_{*}\right)\right. & \in \llbracket\left\langle X,\left[v t_{1}, v t_{2}\right]\right\rangle \rrbracket_{r t *}^{V T, P} \\
& \left.\wedge\left(X, t t, r t_{*}\right) \in \llbracket\left\langle X,\left[t t_{1}, t t_{2}\right]\right\rangle \rrbracket_{r t *}^{T T}\right\} .
\end{aligned}
$$

The definitions show that the framework has been constructed so that the extensionalization of bitemporal tuples is the combination of the extensionalizations for valid and transaction time. It also shows how the reference time $r t_{*}$ serves as an essential coordination mechanism between the valid and transaction time components of the timestamp: the same reference time appears in the valid-time and in the transaction-time denotations. Although it is possible and may be interesting to consider situations where the two reference times differ, we have found that for all practical purposes this coordination is desirable. Nevertheless, other kinds of coordination through the reference time are possible. For example, instead of the standard Cartesian product used here, a coordination mechanism that utilizes a step-wise cross product of the two temporal dimensions is possible [Clifford and Isakowitz 1994].

Another feature of the framework is that the uniform and componentwise treatment of time dimensions makes it easy to include additional dimensions. To specify the semantics of a variable database with additional dimensions, it is necessary to first specify the semantics of the variables and tuples in that new dimension, for example, as is done for the transaction-time dimension in Section 5. Subsequently, the new dimension can be easily integrated with the other dimensions in a definition similar to the previous one. Thus, our framework can be extended to encompass multidimensional temporal databases (also termed indexical [Clifford 1993] and parametric [Gadia and Nair 1993] temporal databases), for example, temporally generalized [Jensen and Snodgrass 1994] and spatiotemporal [AlTaha et al. 1994] databases.

Tables I and II may be combined to cover the bitemporal extensionalizations. The combination of Case $\mathbf{v} \mathbf{1}$ from Table I and Case $\mathbf{t} \mathbf{1}$ from Table II gives the bitemporal extensionalization for a tuple timestamped with a determinate valid time interval, $\left[v t_{1}, v t_{2}\right]$, and a transaction time interval, $\left[t t_{1}, t t_{2}\right]$, both without variables. Note that the transaction time in this case is restricted to the "past" relative to the reference time, just as in transaction-time tuples. For example, the extensionalization at reference time 
June 2 of the tuple

〈Jane, Assistant, [June 3, June 10], [June 1, June 3]〉

is

$\{($ Jane, Assistant, vt, tt, June 2) $\mid v t \in[$ June 3, June 10]

$\wedge t t \in[$ June $1, \min ($ June 2 , June 3$)]\}$.

In this example, the terminating transaction time, June 3 , is constrained by the reference time, June 2.

The graphical representation of bitemporal tuples is three-dimensional; transaction time is the $X$-axis, valid time is the $Y$-axis, and reference time is the $Z$-axis. The reference time has been the $X$-axis, but making the reference time the $Z$-axis in the three-dimensional visualization results in a better picture. The graph is displayed so that the $Z$-axis goes "into" the page. The three-dimensional picture of a bitemporal tuple allows us to represent the passage of time as a spatial displacement, and provides a visual representation for interesting phenomena such as history changes and predictions about the future, as well as incorporating the viewpoint of an observer into these phenomena. As we see in the following, the graphical representation shows the subtle interaction between now, until changed, and the reference time.

Examples of the combinations of the extensionalizations presented in Tables I and II are graphically depicted in Figures 11 and 12. The dotted line vectors in the graph represent directions of growth as the reference time, valid time, or transaction time extends to $T$. Only one generic example tuple is depicted in each case. The evolutionary nature of temporal databases, a key concept, comes through very clearly in the figures. Notice how the shaded areas grow as reference time increases, most prominently for tuples containing variables, indicating an accumulation of knowledge stored in the database. Note also how information in later reference times is always consistent with that in earlier reference times.

Figure 11 illustrates the determinate cases. For example, the lower right corner of Figure 11 depicting the $\mathbf{v} \mathbf{2} \times \mathbf{t} \mathbf{2}$ case shows how now and until changed are bound to an increasing reference time, resulting in a threedimensional stair-shaped pattern. The tuple's extensionalization grows as time passes encompassing more points. In contrast, case $\mathbf{v} \mathbf{1} \times \mathbf{t} \mathbf{1}$ depicts constrained growth, as the tuple ceases to exist beyond transaction time $t t_{2}$. Note that, unless a tuple is known to have been deleted from the database, its "transaction-stop time" is until changed, and hence it has unlimited growth in the transaction-time dimension. This is true for the determinate cases shown in Figure 11 as well as for the indeterminate cases of Figure 12. Notice, for example, how the possible and definite extensionalizations in cases $\mathbf{v 3}^{D} \times \mathbf{t 2}$ and $\mathbf{v 3}^{P} \times \mathbf{t 2}$, the upper right-hand corner of Figure 12, remain constant in the valid-time dimension while growing in transaction- 

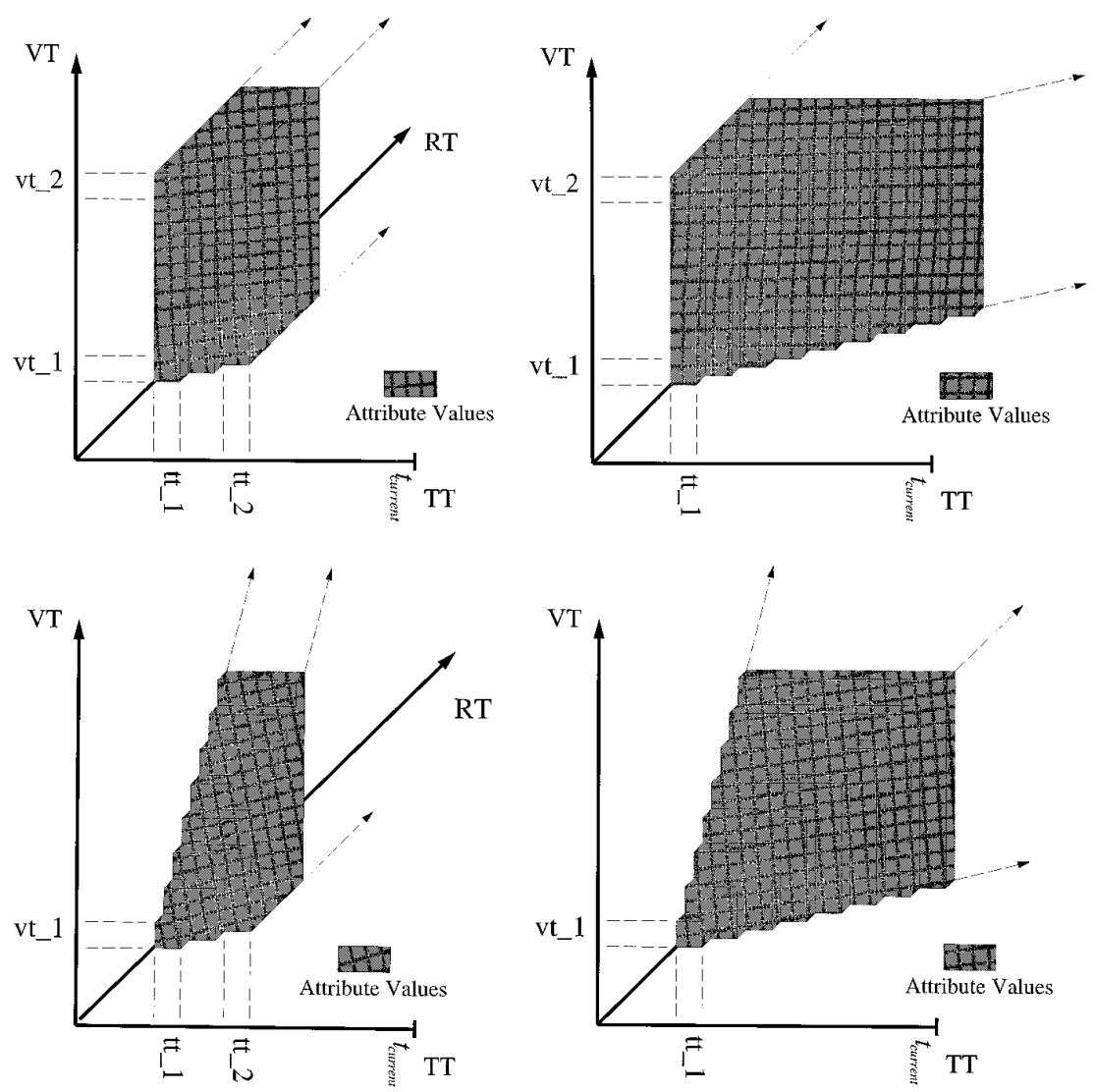

Fig. 11. Examples of bitemporal determinate cases.

time. In contrast, case $\mathbf{v} 4^{D} \times \mathbf{t} 2$ in the lower right-hand corner of Figure 12 , illustrates constrained growth, that is, constant evolution up through time $v t_{2}$.

\subsection{Querying Variable Bitemporal Databases}

The existing bind and timeslice operators, developed for valid-time and transaction-time databases, are easily generalized to apply to bitemporal databases. A bitemporal tuple differs from a valid-time tuple by having a transaction time interval in its timestamp. The valid-time operators are generalized to corresponding bitemporal operators by simply ignoring this extra timestamp. For example, the definite bitemporal valid-time timeslice is defined by generalizing Definition 4.14 as follows.

Definition 6.3 [Variable-Level Definite Bitemporal Valid-Time Timeslice]. Let $S$ be a set of tuples at the variable database level, that is, a set of tuples of the form $T=\left\langle X,\left[v t_{1} \sim v t_{2}, v t_{3} \sim v t_{4}\right],\left[t t_{1}, t t_{2}\right]\right\rangle$, where $T$ is ground. The 

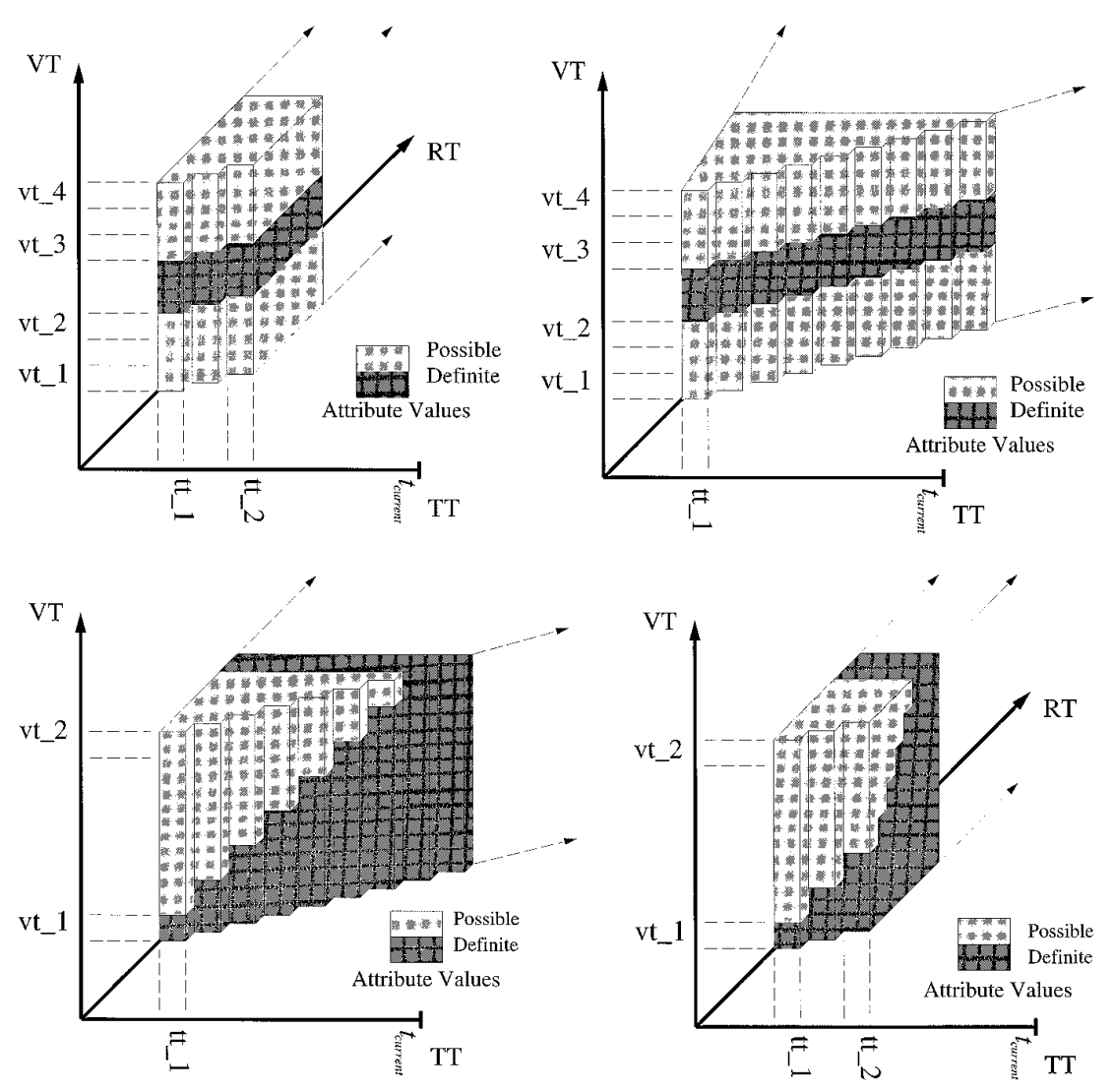

Fig. 12. Examples of bitemporal indeterminate cases.

definite bitemporal valid-time timeslice of $S$ at valid time $v t_{*}$ is defined as follows.

$$
\begin{aligned}
\prod_{v t_{*}}^{D, V, V T, B T}(S) & ={ }_{d f}\left\{\left\langle X,\left[v t_{*}, v t_{*}\right],\left[t t_{1}, t t_{2}\right]\right\rangle \mid\right. \\
T & \left.=\left\langle X,\left[v t_{1} \sim v t_{2}, v t_{3} \sim v t_{4}\right],\left[t t_{1}, t t_{2}\right]\right\rangle \in S\left(v t_{*} \in\left[v t_{2}, v t_{3}\right]\right)\right\} .
\end{aligned}
$$

The superscript " $D, V, V T, B T$ " indicates that the operator considers only the definite information in the tuple, belongs at the variable level, performs a timeslice in the valid-time dimension, and is applicable to bitemporal tuples. In addition to this operator, the subsequent discussion uses the operators $\Pi^{P, V, V T, B T}, \Pi^{V, T T, B T}$, bind $d^{V, V T, B T}$, and bind ${ }^{V, T T, B T}$, which are all similar generalizations of previous definitions.

As with valid-time and transaction-time databases, queries are evaluated by combining bitemporal timeslice and bind operations. Also as before, valid and transaction times must be bound before the bitemporal valid-time timeslice or bitemporal transaction-time timeslice, respectively, can be applied. 


\begin{tabular}{|l|c||c|c|c|c|}
\hline \multicolumn{5}{|c|}{ FACULTY } \\
\hline NAME & RANK & (from) & VALID TIME & \multicolumn{2}{c|}{ TRANS TIME } \\
(start) & (stop) \\
\hline \hline Jane & Assistant & June 1 & (now -3 days) $\sim$ January 1, 2028 & June 2 & until changed \\
\hline
\end{tabular}

Fig. 13. A bitemporal relation.

To explore the interaction of times in queries on bitemporal databases, we consider a number of queries on the simple database depicted in Figure 13 which shows that Jane's employment tuple was added to the database on June 2 . Note that it contains a now-relative indeterminate "to" time and "until changed" as the "stop" time. For the purpose of the example, we assume that today is July 9. Thus, the transaction-time bind operator binds until changed to July 9 in all queries. The first four queries all include Jane in the result.

-Using the current database state, who was possibly a faculty member on July 7 ?

$$
\prod_{\text {July } 7}^{P, V, V T, B T}\left(\operatorname{bind} d_{\text {July } 9}^{V, V T, B T}\left(\prod_{\text {July } 9}^{V, T T, B T}\left(\text { bind }^{V, T T, B T}(\text { Faculty })\right)\right)\right) .
$$

In this query, we transaction timeslice to get only the most current information. The valid-time bind ensures a perspective of today, and the valid timeslice retrieves those tuples that were possibly valid two days ago (on July 7).

-Using the current database state, who was definitely a faculty member on July 1 ?

$$
\left.\prod_{\substack{\text { July } 1 \\ \text { D,VT,BT }}}^{V, V i n d} \operatorname{buly}_{\text {July } 9}^{V, V T, B T}\left(\prod_{\text {July } 9}^{V, T T, B T}\left(\text { bind }^{V, T T, B T}(\text { Faculty })\right)\right)\right) .
$$

As before, the lower bound of the "to" time is ground to July 6 (July $9-3$ days). The difference is solely in the valid timeslice; we require definite information, and so we use a definite timeslice. Since July 1 is before July 6, Jane is in the result.

-Using the database state on July 1 , who was definitely a faculty member on June 15 ?

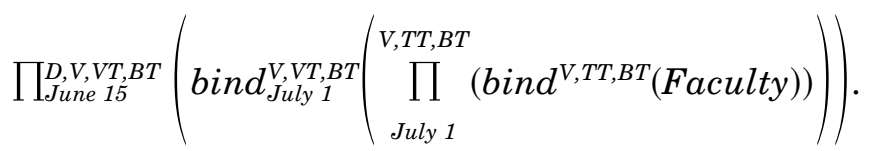

The transaction timeslice retrieves the information current on July 1. The valid-time bind adopts this day as the perspective of the subsequent valid timeslice that retrieves information about June 15. Since Jane's 
tuple was current on July 1 and June 15 is more than three days before July 1 , Jane will be in the result.

-Using the current database state, who will we say on July 12 is possibly a faculty member on September 1 ?

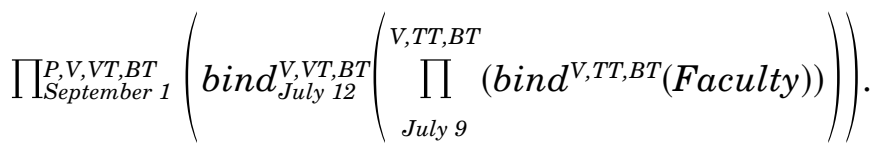

We first consider only current information. Then we adopt a valid-time perspective of July 12 to examine the database as it will appear on that date if no updates are made, that is, our best guess as to what will be current information on July 12. Finally, using that perspective, we ask about possible information on September 1. Jane will be in the result.

In contrast to the preceding queries, the following three queries do not include Jane in the result.

-Using the current database state, who was definitely on the faculty of State University on July 7 ?

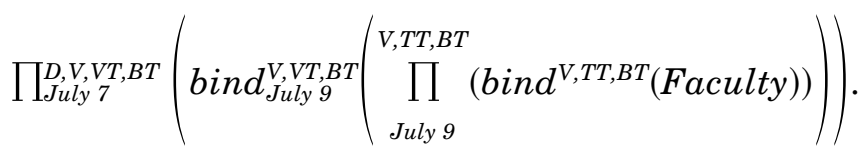

Here, the valid-time bind operation yields a ground "to" time of July 6 January 1, 2028. Since July 7 is after July 6, Jane is possibly, but not definitely, on the faculty.

-Using the database as of July 1 , who was definitely a faculty member on July 1 ?

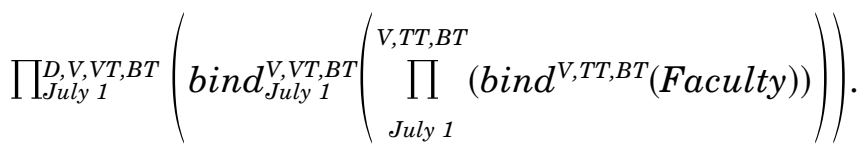

-Using the current database state, who will we say on July 12 is definitely a faculty member on September 1 ?

$$
\prod_{\text {September 1 }}^{D, V, V T, B T}\left(\operatorname{bind}_{\text {July } 12}^{V, V T, B T}\left(\prod_{\text {July } 9}^{V, T T, B T}\left(\text { bind }^{V, T T, B T}(\text { Faculty })\right)\right)\right) .
$$

In most of the preceding examples, the transaction timeslice time and the valid-time bind time, or reference time, are the same. Indeed, this is the typical and most useful scenario, as the following example makes clear. Suppose that today, July 9, we execute a transaction-time timeslice with time argument February 1 (i.e., the preceding February 1). This operation chooses the most up-to-date information as of February 1, and disregards 
information that was not up-to-date on February 1 or was recorded at a later time. The user's perspective for subsequent operations using this information should naturally switch to the frame of reference of the chosen information. Hence, for this example, it would be natural to also bind now to February 1.

Yet, two of the queries previously given illustrate that this is not a necessary restriction. Lifting it leads to increased functionality, but also to queries that are conceptually more involved. Existing query languages generally enforce this restriction.

\section{TIMESTAMP IMPLEMENTATION}

This article has proposed four new current-time-related timestamps, namely, until changed, now, now-relative instants, and now-relative indeterminate instants. Elsewhere we show how these timestamps may be efficiently represented [Dyreson and Snodgrass 1993a, 1993c; Clifford et al. 1994; Dyreson 1994]. For example, a now-relative timestamp can be encoded as a datetime value coupled with a one-bit flag differentiating it from a ground timestamp. Consequently, the timestamps proposed in this article impose little space overhead.

We also proposed adding bind operations for valid time, transaction time, and bitemporal databases; no other operations are needed to support current-time-related modeling entities. The bind operations have no significant impact on the run-time efficiency of a temporal database. The transaction-time bind is very efficient. It simply replaces until changed with the current transaction time. The valid and bitemporal bind operations are only slightly less efficient. For now-relative instants (and nowrelative indeterminate instants) these operations replace now with the reference time and then displace that reference time by a span. The displacement costs one integer addition operation.

Now-relative instants also add an extra comparison to interval constructors. As we observed in Section 2.2.3, predictive updates could insert into the database intervals that end before they start. For a tuple without variables, such intervals can be detected and eliminated when the tuple is first inserted into the database. But a tuple with a variable might initially end before it starts, and only later evolve into a valid interval. Consequently, during run-time each interval involving a variable must be tested to ensure that the starting instant is before the terminating instant. This test needs to be performed only once per interval per query.

\section{SUMMARY AND RESEARCH DIRECTIONS}

The overall conclusion of this article is a recommendation that timestamps involving current-time variables-that is, now, until changed, now-relative, and now-relative indeterminate timestamps-be allowed to be stored as values of columns, for conventional and temporal databases, as well as implicit valid and transaction timestamps, for temporal databases. 
This article makes a number of contributions. First, it provides a formal basis for defining the semantics of databases with variables. The use and generality of the framework was demonstrated by giving a semantics for conventional, valid-time, transaction-time, and bitemporal databases with all existing variables. Apart from specifying a reasonable semantics for such databases, this exercise demonstrates important properties of the framework. The first property is that it is capable of capturing the semantics of a wide range of variables. We provide the semantics of variables of all kinds of general temporal aspects of database facts currently identified [Jensen et al. 1994a]: user-defined time, valid time, and transaction time. The second is that the semantics of a multidimensional database may be specified as a coordinated combination of the semantics of the constituent one-dimensional databases. The reference-time dimension in the framework provides the coordination mechanism. For example, the semantics of variable bitemporal databases was specified very easily by using the already specified semantics for valid-time and transaction-time databases. This property makes it relatively easy to specify the semantics of multidimensional databases. It also makes it easy to add further kinds of time that may emerge in the future, as well as other dimensions, such as space, again, in all their various combinations.

Second, without current-time variables, temporal databases provide inadequate support for their applications. The article demonstrates that existing variables, such as now and until changed, are indispensable in databases. It also identifies situations where even these variables are inadequate, and introduces new now-relative and now-relative indeterminate instants that provide the desired support. The semantics of databases with such variables is also defined within the framework.

Third, a foundation for the querying of variable databases from existing temporal query languages was presented. The article provides algebraic "bind" operators for valid-time, transaction-time, and bitemporal databases, and it shows how these can be used to permit existing query languages to access variable databases. As a first step during query processing, the bind operation is applied to variable databases, thus replacing all variables with ground values appropriate for the processing of the query at hand. The framework also clarifies which values are the appropriate ground values. This approach encapsulates the handling of variables in a single operator per temporal dimension. It also requires only minimal changes to the query processor: support for one new operator has to be added; all other components remain unchanged.

These three observations provide the rationale for the conclusion that variable databases are viable. A number of secondary, but noteworthy, contributions also deserve mention. The article resolves the meaning of the use of variables in existing temporal data models. A graphical notation with two or three dimensions used throughout the article proved to be helpful when describing the semantics of variable databases. The complex interactions of current time, reference time, transaction time, and valid time within queries and variable databases were investigated in detail. 
These interactions were not thoroughly understood or explicated in existing bitemporal data models. The concept of "perspective" within queries was illustrated. Perspective adds the ability to bind the valid-time variable now to times other than the current time. Supporting this notion within a query language enhances its functionality when querying variable databases.

This framework has implications for database query language design. The user-defined time types available in SQL-92 can be easily extended to store now-relative and indeterminate nonrelative variables as values in columns. The TSQL2 language [Snodgrass 1995] does so, and also supports those variables for valid and transaction time. In TSQL2 the "bind" operation is implicit; NOBIND is provided to store variables in the database.

There are several directions for future research. The precise semantics of several temporal models proposed in the literature could profitably be examined in light of the framework presented here. In defining the semantics for bitemporal databases, we have chosen but one possible way of combining the semantics of valid-time and transaction-time databases; other possible combinations of these two temporal dimensions might also prove useful. In addition, the use of the graphical representation of temporal relations at the user interface-for displaying the results of queries and, for example, for the assertion of temporal integrity constraints-seems to us a promising one for further research. The impact of stored variables on database storage structures and access methods is an open problem. It also presents an opportunity, for example, if the optimizer knows (through attribute statistics) that a large proportion of tuples have a "to" time of now. The optimizer may then decide that a sort-merge temporal join will be less effective. Finally, new kinds of variables, such as here for spatial and spatiotemporal databases, should be investigated, as an extension of the framework introduced in this article.

\section{ACKNOWLEDGMENTS}

Won Kim, Nick Kline, and the anonymous referees provided helpful comments on a previous draft.

\section{REFERENCES}

Al-Taha, K. K., Snodgrass, R. T., And Soo, M. D. 1994. Bibliography on spatiotemporal databases. Int. J. Geograph. Inf. Syst. 8, 1 (Jan.-Feb.), 95-103.

Ariav, G., Beller, A., ANd Morgan, H. L. 1984. A temporal data model. Tech. Rep. DS-WP 82-12-05, Decision Sciences Dept., Univ. of Pennsylvania, Dec.

BAssiouni, M. A. AND Llewellyn, M. J. 1992. A relational-calculus query language for historical databases. Comput. Lang. 17, 3, 185-197.

Ben-Zvi, J. 1982. The time relational model. Univ. of California at Los Angeles, Ph.D. Thesis.

Bhargava, G. AND GadiA, S. 1989. Achieving zero information loss in a classical database environment. In Proceedings of the International Conference on Very Large Databases (Amsterdam, Aug.), 217-224.

Brusoni, V., Console, L., Terenziani, P., And Pernici, B. 1995. Extending temporal relational databases to deal with imprecise and qualitative temporal information. In Proceedings of the VLDB International Workshop on Temporal Databases (Zurich, Switzerland, 
Sept.), J. Clifford and A. Tuzhilin (Eds), Workshops in Computing Series, Springer Verlag, New York, NY, 3-22.

Clifford, J. 1993. Indexical databases. In Advanced Database Systems, Lecture Notes in Computer Science 759, Springer-Verlag.

Clifford, J. AND CROKer, A. 1987. The historical relational data model HRDM and algebra based on lifespans. In Proceedings of the IEEE International Conference on Data Engineering (Los Angeles, CA, Feb.), 528-537.

Clifford, J., Croker, A., AND Tuzhilin, A. 1993. On completeness of historical relational query languages. ACM Trans. Database Syst. 19, 2 (March), 64-116.

Clifford, J., Dyreson, C. E., Isakowitz, T., Jensen, C. C., And Snodgrass, R. T. 1994. On the semantics of 'now' in temporal databases. Tech. Rep. R-94-2047, Aalborg Univ., Dept. of Mathematics and Computer Science, Denmark, Nov.

Clifford, J. AND Isakowitz, T. 1993. On the semantics of transaction time and valid time in bitemporal databases. In Proceedings of the ARPA/NSF International Workshop on an Infrastructure for Temporal Databases (Arlington, TX, June), R. T. Snodgrass, Ed., I.1-I.17.

Clifford, J. AND IsAKowitz, T. 1994. On the semantics of (bi)temporal variable databases. In Proceedings of the Fourth International Conference on Extending Database Technology (Cambridge, England, March), 215-230.

Clifford, J. AND TANsel, A. U. 1985. On an algebra for historical relational databases: Two views. In Proceedings of ACM SIGMOD International Conference on Management of Data, S. Navathe, Ed., (Austin, TX, May), 247-265.

Clifford, J. AND WARREN, D. S. 1983. Formal semantics for time in databases. ACM Trans. Database Syst. 8, 2, 214-254.

CodD, E. F. 1970. A relational model of data for large shared data banks. Commun. ACM 13, 6 (June), 377-387.

Date, C. J. And White, C. J. 1990. A Guide to DB2, Vol. 1, 3rd ed. Addison-Wesley, Reading, MA.

Dutta, S. 1989. Generalized events in temporal databases. In Proceedings of the Fifth International Conference on Data Engineering, (Los Angeles, CA, Feb.), 118-126.

Dyreson, C. E. 1994. Valid-time indeterminacy. Computer Science Dept., Univ. of Arizona. Ph.D. Thesis, Oct.

Dyreson, C. E. AND Snodgrass, R. T. 1993a. Timestamp semantics and representation. Inf. Syst. 18, 3, 143-166.

Dyreson, C. E. And Snodgrass, R. T. 1993b. Valid-time indeterminacy. In Proceedings of the International Conference on Data Engineering (Vienna, Austria, April), 335-343.

Dyreson, C. E. AND Snodgrass, R. T. 1993c. A timestamp representation. In The TSQL Temporal Query Language, R. T. Snodgrass, Ed., Kluwer, Boston, MA, 1995, Chap. 25, 475-499.

ElmasRI, R., WuU, G., AND KIm, Y. 1990. The time index-an access structure for temporal data. In Proceedings of the International Conference on Very Large Data Bases (Brisbane, Australia, Aug.), 1-12.

Finger, M. 1992. Handling database updates in two-dimensional temporal logic. J. Appl. Non-Classical Logics 2, 2.

Fraenkel, A. A., Bar-Hillel, Y., And Levy, A. 1973. Foundations of Set Theory. NorthHolland, Amsterdam, Netherlands.

GADIA, S. K. 1988. A homogeneous relational model and query languages for temporal databases. ACM Trans. Database Syst. 13, 4 (Dec.), 418-448.

GADIA, S. AND NAIR, S. 1993. Temporal databases: A prelude to parametric data. In Temporal Databases: Theory, Design, and Implementation, A. Tansel et al., Eds., Benjamin Cummings, Redwood City, CA, Chap. 2, 28-66.

GADIA, S. K., NAIR, S., AND Poon, Y.-C. 1992. Incomplete information in relational temporal databases. In Proceedings of the Conference on Very Large Data Bases (Vancouver, Canada, Aug.).

Jensen, C. S. And Mark, L. 1990. A framework for vacuuming temporal databases. Tech. Rep. CS-TR-2516/UMIACS-TR-90-105, Univ. of Maryland, Dept. of Computer Science, College Park, MD, Aug. 
Jensen, C. S. AND MaRK, L. 1992. Queries on change in an extended relational model. IEEE Trans. Knowl. Data Eng. 4, 2 (April), 192-200.

Jensen, C. S. and Snodgrass, R. T. 1992. Temporal specialization. In Proceedings of the IEEE International Conference on Data Engineering (Tempe, AZ, Feb.), 594-603.

Jensen, C. S. And Snodgrass, R. T. 1994. Temporal specialization and generalization. IEEE Trans. Knowl. Data Eng. 6, 6 (Dec.), 954-974.

Jensen, C. S. And Snodgrass, R. T. 1996. Semantics of time-varying information. Inf. Syst. $21,4,311-352$.

Jensen, C. S., Clifford, J., Elmasri, R., Gadia, S. K., Hayes, P., And Jajodia, S. (Eds.) 1994. A consensus glossary of temporal database concepts. ACM SIGMOD Rec., 23, 1 (March), $52-65$.

Jensen, C. S., Soo, M. D., And Snodgrass, R. T. 1994 . Unifying temporal data models via a conceptual model. Inf. Syst. 197 (Dec.), 513-547.

Kurutach, W. AND FrankLin, J. 1993. On temporal-fuzziness in temporal fuzzy databases. In DEXA'93 (Prague, Czech Republic, Sept.), 154-165.

LIPSKI, W. JR. 1979. On semantic issues connected with incomplete information databases. ACM Trans. Database Syst. 4, 3 (Sept.), 262-296.

Lorentzos, N. A. And Johnson, R. G. 1988. Extending relational algebra to manipulate temporal data. Inf. Syst. 13, 3, 286-296.

Melton, J. AND Simon, A. R. 1993. Understanding the New SQL: A Complete Guide. Morgan-Kaufmann, San Mateo, CA.

Montague, R. 1974. Formal Philosophy: Selected Papers of Richard Montague. Yale University Press, New Haven, CT.

Navathe, S. B. And Ahmed, R. 1989. A temporal relational model and a query language. Inf. Sci. 49, 147-175.

REITER, R. 1984. Towards a logical reconstruction of relational database theory. In On Conceptual Modelling, Springer Verlag, New York, 191-233.

RodDICK, J. F. 1992. Schema evolution in database systems-An annotated bibliography. SIGMOD Rec. 21, 4 (Dec.), 35-40.

SARDA, N. L. 1990. Algebra and query language for a historical data model. Computer J., 33, 1 (Feb.), 11-18.

Snodgrass, R. T. 1987. The temporal query language TQuel. ACM Trans. Database Syst. 12, 2 (June), 247-298.

Snodgrass, R. T. 1993. An overview of TQuel. In Temporal Databases: Theory, Design, and Implementation, A. Tansel et al., Eds., Benjamin-Cummings, Redwood City, CA, Chap. 6, 141-182.

SNOdGrass, R. T. (ED.) 1995. The TSQL2 Temporal Query Language. Kluwer, Boston, MA.

Snodgrass, R. T. AND Ahn, I. 1985. A taxonomy of time in databases. In Proceedings of ACM SIGMOD International Conference on Management of Data (Austin, TX, May), 236246.

Sykes, J. B. (ED.) 1964. The Concise Oxford Dictionary. Oxford University Press, Oxford, England.

Tansel, A. U. 1990. Modelling temporal data. Inf. Sofw. Technol. 32, 8 (Oct.), 514-520.

Tansel, A., Clifford, J., Gadia, S., Jajodia, S., Segev, A., And Snodgrass, R. T. (Eds.) 1993. Temporal Databases: Theory, Design, and Implementation. Benjamin-Cummings, Redwood City, CA.

Thirumalai, S. And Krishna, S. 1988. Data organization for temporal databases. Tech. Rep., Raman Research Institute, Bangalore, India.

Wiederhold, G., JAJOdiA, S., AND Litwin, W. 1993. Integrating temporal data in a heterogeneous environment. In Temporal Databases: Theory, Design, and Implementation. Benjamin-Cummings, Redwood City, CA, 563-579.

YAU, C. AND Chat, G. S. W. 1991. TempSQL-A language interface to a temporal relational model. Inf. Sci. Tech. (Oct.), 44-60.

Received November 1994; revised August 1996; accepted September 1996 